\title{
Existence and nonexistence of solutions for the heat equation with a superlinear source term
}

\author{
Yohei Fujishima* \\ Department of Mathematical and Systems Engineering \\ Faculty of Engineering, Shizuoka University \\ 3-5-1 Johoku, Hamamatsu 432-8561, Japan \\ Norisuke Ioku ${ }^{\dagger}$ \\ Graduate School of Science and Engineering, Ehime University \\ Matsuyama, Ehime 790-8577, Japan
}

\begin{abstract}
Classification theory on the existence and non-existence of local in time solutions for initial value problems of nonlinear heat equations are investigated. Without assuming a concrete growth rate on a nonlinear term, we reveal the threshold integrability of initial data which classify existence and nonexistence of solutions via a quasi-scaling and its invariant integral. Typical nonlinear terms, for instance polynomial type, exponential type and its sum, product and composition, can be treated as applications.
\end{abstract}

Keywords: nonlinear heat equation, scale invariance, singular initial data, existence and nonexistence

2010 MSC: Primary; 35K55, Secondly; 35A01, 46E30

\section{Introduction}

We consider existence and nonexistence of solutions for a heat equation with general nonlinearity

$$
\begin{cases}\partial_{t} u=\Delta u+f(u) \quad \text { in } & \mathbb{R}^{N} \times(0, T), \\ u(x, 0)=u_{0}(x) \geq 0 & \text { in } \quad \mathbb{R}^{N}\end{cases}
$$

where $\partial_{t}=\partial / \partial t, N \geq 1, T>0, u_{0}$ is a nonnegative measurable initial function and $f \in$ $C^{1}([0, \infty))$ is a positive monotonically increasing function in $(0, \infty)$, that is,

$$
f(s)>0, \quad f^{\prime}(s)>0 \text { for all } s \in(0, \infty) .
$$

In the following, for suitable Banach space $X$, we say a function $u \in C^{2,1}\left(\mathbb{R}^{N} \times(0, T)\right)$ is a classical solution in $X$ for problem (1.1) if $u$ satisfies the equation in the classical sense and $\left\|u(t)-e^{t \Delta} u_{0}\right\|_{X} \rightarrow 0$ as $t \rightarrow 0$, where $e^{t \Delta} u_{0}$ denotes the solution of the heat equation with the initial data $u_{0}$.

It follows from the standard argument that problem (1.1) possesses the unique classical solution in $L^{\infty}\left(\mathbb{R}^{N}\right)$ for general nonlinearities $f \in C^{1}([0, \infty))$ if $u_{0} \in L^{\infty}\left(\mathbb{R}^{N}\right)$. On the other

\footnotetext{
*e-mail address: fujishima@shizuoka.ac.jp
}

${ }^{\dagger}$ e-mail address: ioku@ehime-u.ac.jp 
hand, for the case $u_{0} \notin L^{\infty}\left(\mathbb{R}^{N}\right)$, existence results of solutions for problem (1.1) heavily depend on the growth rate of the nonlinear term $f$. One of the typical examples of $f$ is a power type nonlinearity, that is,

$$
\partial_{t} u=\Delta u+u^{p}, \quad x \in \mathbb{R}^{N}, t>0, \quad u(x, 0)=u_{0}(x) \geq 0, \quad x \in \mathbb{R}^{N},
$$

where $p>1$. This equation for unbounded initial data has been studied intensively since the pioneering work due to Weissler [26], and there hold the following:

- if $r \geq \frac{N}{2}(p-1)$ and $r>1$ or $r>\frac{N}{2}(p-1)$ and $r \geq 1$, then for any $u_{0} \in L^{r}\left(\mathbb{R}^{N}\right)$, there exist a constant $T>0$ and a local in time classical solution $u \in C\left([0, T] ; L^{r}\left(\mathbb{R}^{N}\right)\right)$ for problem (1.3).

- if $\frac{N}{2}(p-1)>1$ and $1 \leq r<\frac{N}{2}(p-1)$, then there exists an initial function $u_{0} \in L^{r}\left(\mathbb{R}^{N}\right)$ such that problem (1.3) can not possess any local in time nonnegative classical solutions.

See [1], 3], 44, [6]-[12], [15], [18, 20]-[26] for existence and nonexistence of solutions for nonlinear parabolic equations and qualitative properties of solutions. We also refer to [14 and [19], which include good references concerning parabolic equations. It is remarkable that the critical exponent $r_{c}:=\frac{N}{2}(p-1)$, which gives the classification of existence and nonexistence of solutions for problem (1.3), arises from the scale invariant property of (1.3) under the scaling transformation

$$
u_{\lambda}(x, t):=\lambda^{\frac{2}{p-1}} u\left(\lambda x, \lambda^{2} t\right), \quad \lambda>0,
$$

in the sense that $\left\|u_{\lambda}(\cdot, 0)\right\|_{L^{r}\left(\mathbb{R}^{N}\right)}=\|u(\cdot, 0)\|_{L^{r}\left(\mathbb{R}^{N}\right)}$ if and only if $r=r_{c}$. The above assertions due to Weissler show that the critical exponent $r=r_{c}$ can be a priori found by the scaling transformation and its invariant norm of problem (1.3). However, in the case of general nonlinearity $f(u)$, it is not clear which integral should be controlled for the classification of existence and nonexistence of solutions, since problem (1.1) does not possess a scale invariant property for general nonlinearity $f$.

In this paper, we reveal the threshold integrability of $u_{0}$ to classify existence and nonexistence of solutions for problem (1.1) without any concrete assumptions on the behavior of $f=f(s)$ near $s=\infty$. To this end, we apply the "quasi" scaling proposed by the first author of this paper in [5]:

$$
u_{\lambda}(x, t):=F^{-1}\left[\lambda^{-2} F\left(u\left(\lambda x, \lambda^{2} t\right)\right)\right], \quad \lambda>0,
$$

where

$$
F(s):=\int_{s}^{\infty} \frac{d u}{f(u)}
$$

and $F^{-1}$ is the inverse function of $F$. We mention that the transformation (1.5) does not preserve the equation (1.1), however, the main term of (1.1) are unchanged. In fact, for the solution $u$ of (1.1), the function $u_{\lambda}$ defined by (1.5) satisfies

$$
\partial_{t} u_{\lambda}=\Delta u_{\lambda}+f\left(u_{\lambda}\right)+f\left(u_{\lambda}\right) f(u)^{-2} F(u)^{-1}|\nabla u|^{2}\left[f^{\prime}(u) F(u)-f^{\prime}\left(u_{\lambda}\right) F\left(u_{\lambda}\right)\right] .
$$

It should be emphasized that this striking transformation (1.5) is a generalization of (1.4) for polynomial nonlinearity since (1.5) coincides with (1.4) if $f(u)=u^{p}$ and the remainder term in (1.6) becomes zero.

We now introduce a significant property which arises from the transformation (1.5), that is, the scale invariant property

$$
\int_{\mathbb{R}^{N}} F\left(u_{\lambda}(x, 0)\right)^{-\frac{N}{2}} d x=\int_{\mathbb{R}^{N}} F(u(x, 0))^{-\frac{N}{2}} d x, \quad \lambda>0 .
$$


This property plays an essential role for classifying existence and nonexistence as $L^{r_{c}}$ norm for problem (1.4). Furthermore, we focus on the limit of $f^{\prime}(s) F(s)$ as $s \rightarrow \infty$ :

$$
A:=\lim _{s \rightarrow \infty} f^{\prime}(s) F(s),
$$

since the behavior of the function $f^{\prime} F$ controls the remainder term of (1.6). Throughout this paper, we assume that the above limit $A$ always exists and $f$ is superlinear in the sense that

$$
F(s)=\int_{s}^{\infty} \frac{d u}{f(u)}<\infty
$$

for all $s>0$. Note that the limit $A$ always exist for typical examples of $f$ such as

$$
f(u)=u^{p}(p>1), \quad u^{p}+u^{q}(p>q>1), \quad e^{u}, \quad e^{u^{2}} .
$$

The main purpose of this paper is to show that the integrability driven from (1.7) implies the classification theorems of existence of solutions for problem (1.1) with general nonlinearity $f$. To state the results, we introduce some notation. For $x \in \mathbb{R}^{N}$ and $\rho>0$, we denote by $B_{\rho}(x)$ the ball of radius $\rho$ centered at $x$. For $1 \leq p<\infty$, define the uniformly local $L^{p}$ space $L_{u l, \rho}^{p}\left(\mathbb{R}^{N}\right)$ by

$$
L_{u l, \rho}^{p}\left(\mathbb{R}^{N}\right):=\left\{u \in L_{l o c}^{p}\left(\mathbb{R}^{N}\right):\|u\|_{L_{u l, \rho}^{p}\left(\mathbb{R}^{N}\right)}:=\sup _{y \in \mathbb{R}^{N}}\left(\int_{B_{\rho}(y)}|u(x)|^{p} d x\right)^{1 / p}<\infty\right\} .
$$

We denote by $\mathcal{L}_{u l, \rho}^{p}\left(\mathbb{R}^{N}\right)$ the closure of the space of bounded uniformly continuous functions $B U C\left(\mathbb{R}^{N}\right)$ in the space $L_{u l, \rho}^{p}\left(\mathbb{R}^{N}\right)$, that is,

$$
\left.\mathcal{L}_{u l, \rho}^{p}\left(\mathbb{R}^{N}\right):=\overline{B U C\left(\mathbb{R}^{N}\right)}\|\cdot\|_{L_{u l, \rho}^{p}} \mathbb{R}^{N}\right) .
$$

Define

$$
\left(e^{t \Delta} \varphi\right)(x):=(4 \pi t)^{-\frac{N}{2}} \int_{\mathbb{R}^{N}} e^{-\frac{|x-y|^{2}}{4 t}} \varphi(y) d y
$$

for $\varphi \in L_{u l, \rho}^{1}\left(\mathbb{R}^{N}\right)$. Then $e^{t \Delta} \varphi$ gives the solution for the heat equation with the initial data $\varphi$.

We are ready to state our main results. We first state the existence results of local in time solutions for problem (1.1).

Theorem 1.1. Let $N \geq 1, \rho>0$ and $f \in C^{1}([0, \infty))$ satisfy (1.2) and (1.8). Assume that the limit $A=\lim _{s \rightarrow \infty} f^{\prime}(s) F(s)$ exists and that there exists a constant $s_{1}>0$ such that

$$
f^{\prime}(s) F(s) \leq A \text { for all } s \geq s_{1} .
$$

(i) (Subcritical case) Let $r>0$ be a constant satisfying

$$
r \geq A-1, \quad r>\frac{N}{2}
$$

and assume that a nonnegative initial function $u_{0}$ is measurable and satisfies

$$
F\left(u_{0}\right)^{-r} \in L_{u l, \rho}^{1}\left(\mathbb{R}^{N}\right) .
$$

- If $A>1$, there exist $T>0$ and a local in time classical solution $u \in C^{2,1}\left(\mathbb{R}^{N} \times(0, T)\right)$ of (1.1) satisfying the initial value problem in the following sense:

$$
\lim _{t \rightarrow 0}\left\|u(t)-e^{t \Delta} u_{0}\right\|_{L_{u l, \rho}^{\frac{r}{A-1}}\left(\mathbb{R}^{N}\right)}=0 .
$$


Furthermore, the existence time $T$ can be estimated to satisfy

$$
\begin{aligned}
& T^{\frac{N}{2}\left(1-\frac{1}{A}\right)} \rho^{-N\left(1-\frac{1}{A}\right)} \\
& \quad+\max \left\{\left\|F\left(u_{0}\right)^{-r}\right\|_{L_{u l, \rho}^{1}\left(\mathbb{R}^{N}\right)}, F\left(s_{1}\right)^{-r} \rho^{N}\right\}\left(T^{r-\frac{N}{2 A}} \rho^{-N \cdot \frac{A-1}{A}}+T^{r-\frac{N}{2}}\right) \geq \gamma
\end{aligned}
$$

where $\gamma>0$ depends only on $N, A$ and $r$.

- If $A=1$, there exist $T>0$ and a local in time classical solution $u \in C^{2,1}\left(\mathbb{R}^{N} \times(0, T)\right)$ of (1.1) satisfying the initial value problem in the following sense:

$$
\lim _{t \rightarrow 0}\left\|u(t)-e^{t \Delta} u_{0}\right\|_{L^{\infty}\left(\mathbb{R}^{N}\right)}=0
$$

Furthermore, the existence time $T$ can be estimated to satisfy

$$
\begin{aligned}
& T^{\frac{\epsilon N}{2}} \rho^{-\epsilon N} \\
& +\gamma_{\epsilon} \max \left\{\left\|F\left(u_{0}\right)^{-r}\right\|_{L_{u l, \rho}^{1}\left(\mathbb{R}^{N}\right)}, F\left(s_{1}\right)^{-r} \rho^{N}\right\}\left(T^{r-\frac{N}{2}(1-\epsilon)} \rho^{-\epsilon N}+T^{r-\frac{N}{2}}\right) \geq \gamma
\end{aligned}
$$

for any sufficiently small $\epsilon>0$, where $\gamma$ depends only on $N$ and $r$, and $\gamma_{\epsilon}$ is a positive constant depending only on $N, r$ and $\epsilon$ satisfying $\gamma_{\epsilon} \rightarrow \infty$ as $\epsilon \rightarrow 0$.

(ii) (Critical case) Let

$$
r=\frac{N}{2}>A-1,
$$

and assume that a nonnegative initial function $u_{0}$ is measurable and satisfies

$$
F\left(u_{0}\right)^{-r} \in \mathcal{L}_{u l, \rho}^{1}\left(\mathbb{R}^{N}\right) .
$$

Then there exist $T>0$ and a local in time classical solution $u \in C^{2,1}\left(\mathbb{R}^{N} \times(0, T)\right)$ of (1.1) satisfying (1.12) for the case $A>1$ and (1.14) for the case $A=1$.

In view of Theorem 1.1, the balance of two important factors, the behavior of the function $f=f(s)$ as $s \rightarrow \infty$ and the singularity of $u_{0}$ (given in (1.11) or (1.17)), is controlled by the constant $A=\lim _{s \rightarrow \infty} f^{\prime}(s) F(s)$ via the function $f$. Note that it relies on the setting of $f$ and the space of the initial data whether the solution $u(t)$ converges to $u_{0}$ as $t \rightarrow 0$, so we do not discuss this problem under general setting of Theorem 1.1. However, we consider the convergence of the solution to the initial data for concrete examples of $f$ in Section [5. See also Remark 1.3.

Remark 1.1. It must hold that $A \geq 1$ for all superlinear functions $f \in C^{1}([0, \infty))$ satisfying (1.2) and (1.8). Assume that $A<1$. Then there exist $s_{0}>0$ and $\epsilon \in(0,1)$ such that $f^{\prime}(s) F(s) \leq$ $1-\epsilon$ for all $s \geq s_{0}$. Since $f^{\prime}(s)=F^{\prime \prime}(s) / F^{\prime}(s)^{2}$ and $F^{\prime}(s)=-1 / f(s)<0$, it holds

$$
\frac{F^{\prime \prime}(s)}{F^{\prime}(s)} \geq(1-\epsilon) \frac{F^{\prime}(s)}{F(s)}
$$

for all $s \geq s_{0}$. Integrating both sides of above inequality on $\left(s_{0}, s\right)$, we have

$$
-F^{\prime}(s) \geq C F(s)^{1-\epsilon},
$$

where $C>0$ is a constant. This together with a simple calculation contradicts the positivity of F. 
Remark 1.2. One can easily check that

$$
\lim _{s \rightarrow \infty} f^{\prime}(s) F(s)=\left\{\begin{array}{cl}
\frac{p}{p-1} & \text { if } f(u)=u^{p} \text { with } p>1, \\
1 & \text { if } f(u)=e^{u} \text { or } f(u)=e^{u^{2}} .
\end{array}\right.
$$

Therefore the constant $A$ becomes small if the growth rate of $f$ is rapid.

Remark 1.3. Under the condition (1.9), as in Remark 1.1, we have

$$
\frac{F^{\prime \prime}(s)}{F^{\prime}(s)} \geq A \frac{F^{\prime}(s)}{F(s)}
$$

which implies that $-F^{\prime}(s) \geq C F(s)^{A}$ for all sufficiently large $s>0$. Here $C>0$ is a constant independent of $s$. Then, by a simple calculation we can check that $s \leq C F(s)^{-(A-1)}$ for some $C>0$ and all sufficiently large $s>0$ and that $F\left(u_{0}\right)^{-r} \in L_{u l, \rho}^{1}\left(\mathbb{R}^{N}\right)$ yields $u_{0} \in L_{u l, \rho}^{\frac{r}{A-1}}\left(\mathbb{R}^{N}\right)$ for the case $A>1$ provided that either (1.10) and (1.11) or (1.16) and (1.17) hold. Therefore, the convergence (1.12) of $u(t)$ is reasonable from the viewpoint of the singularity of $u_{0}$. On the other hand, (1.11) does not imply that $u_{0} \in \mathcal{L}_{u l, \rho}^{\frac{N}{2} \cdot \frac{1}{A-1}}\left(\mathbb{R}^{N}\right)$ for the case $A>1$ in general. So we do not know whether $e^{t \Delta} u_{0}$ can be replaced by $u_{0}$ in (1.12). See also Lemma 2.2.

We next state the nonexistence results of local in time solutions for problem (1.1).

Theorem 1.2 (Nonexistence). Let $N \geq 1, \rho>0$ and $f \in C^{1}([0, \infty))$ satisfy (1.2) and (1.8). Assume that the limit $A=\lim _{s \rightarrow \infty} f^{\prime}(s) F(s)$ exists. Furthermore, assume that $A-1<N / 2$ and there exists a constant $s_{2}>0$ such that

$$
f^{\prime}(s) F(s) \geq A \text { for all } s \geq s_{2} .
$$

Then, for any $r \in[A-1, N / 2)$ if $A>1$ or any $r \in(0, N / 2)$ if $A=1$, there exists a nonnegative measurable initial function $u_{0}$ satisfying $F\left(u_{0}\right)^{-r} \in L_{u l, \rho}^{1}\left(\mathbb{R}^{N}\right)$ such that there can not exist nonnegative classical solutions of (1.1) satisfying the initial value problem in the sense (1.12) or (1.14).

In some examples of $f$, for instance $f(u)=u^{p}+u^{q}(p>q>1)$, the condition (1.18) does not hold. However, for the case $A>1$, it seems possible to avoid this difficulty by considering some approximation of $f$ from above (See Theorem 5.1 and its proof). On the other hand, for the case $A=1$, similar calculations as in Remark 1.3 can be carried out, and the condition (1.18) with $A=1$ implies that there exists a constant $C>0$ such that $f(u) \leq e^{C u}$. Therefore, Theorem 1.2 is no longer available for rapidly growing nonlinearity such as $f(u)=e^{u^{2}}$. However, even for the case $f$ has rapid growth, that is, $A=1$ and $f^{\prime}(s) F(s)$ converges to 1 from below, there holds the following nonexistence result.

Theorem 1.3 (Nonexistence). Let $N \geq 1, \rho>0$ and $f \in C^{1}([0, \infty))$ satisfy (1.2) and (1.8). Assume that $A=1$ and there exists a constant $s_{3}>0$ such that

$$
f^{\prime}(s) F(s) \leq 1 \text { for all } s \geq s_{3} .
$$

Then, for any $r \in(0, N / 2)$, there exists a nonnegative measurable initial function $u_{0}$ satisfying $F\left(u_{0}\right)^{-r} \in L_{u l, \rho}^{1}\left(\mathbb{R}^{N}\right)$ such that there can not exist nonnegative classical solutions of (1.1) satisfying the initial value problem in the sense (1.14).

So far as the authors know, these are the first results to characterize existence and nonexistence of solutions for problem (1.1) without any assumptions on the growth rate of the nonlinear term $f$. 
As an application of our main results, we treat the case $f(u)=e^{u^{2}}$. Several other applications are considered in Section 5 , Consider

$$
\begin{cases}\partial_{t} u=\Delta u+e^{u^{2}}, & x \in \mathbb{R}^{N}, t>0, \\ u(x, 0)=u_{0}(x) \geq 0, & x \in \mathbb{R}^{N} .\end{cases}
$$

This type nonlinearity appears in view of the Trudinger-Moser inequality or the problem related to uniqueness results for (1.3) with $N=2$, and has been treated in [9, [10, [11] and [21. See also [17. Among others, in [10], [11] and [21, the authors discuss local in time existence and nonexistence of solutions for (1.19) (with a slight modification on the nonlinearity) in the Orlicz space $\exp L^{2}\left(\mathbb{R}^{N}\right)$. Here the Orlicz space $\exp L^{2}\left(\mathbb{R}^{N}\right)$ is the set of all functions $u_{0} \in L_{\text {loc }}^{1}\left(\mathbb{R}^{N}\right)$ satisfying

$$
\int_{\mathbb{R}^{N}}\left[e^{\lambda\left|u_{0}(x)\right|^{2}}-1\right] d x<\infty
$$

for some $\lambda>0$. Local in time existence of solutions for problem (1.19) has been shown for initial data $u_{0}$ satisfying (1.20) with sufficiently large $\lambda>0$, and they also prove that (1.19) can not possess local in time solutions for some $u_{0}$ satisfying (1.20) with sufficiently small $\lambda>0$. It seems that the critical integrability for $u_{0}$ which classifies local in time existence and nonexistence of solutions for (1.19) is not known yet. In the following theorem, we apply Theorems 1.1 and 1.3 to obtain the critical integrability of initial data $u_{0}$.

Theorem 1.4. Let $N \geq 1$ and $\rho>0$.

(i) (Subcritical case) Let $r>N / 2$. For any nonnegative measurable function $u_{0}$ satisfying $\left|u_{0}\right|^{r} e^{r\left|u_{0}\right|^{2}} \in L_{u l, \rho}^{1}\left(\mathbb{R}^{N}\right)$, there exists a local in time classical solution for problem (1.19) satisfying (1.14). Furthermore, if $\left|u_{0}\right|^{r} e^{r\left|u_{0}\right|^{2}} \in \mathcal{L}_{u l, \rho}^{1}\left(\mathbb{R}^{N}\right)$, then

$$
\lim _{t \rightarrow 0} \sup _{y \in \mathbb{R}^{N}} \int_{B_{\rho}(y)}\left|u(x, t)-u_{0}(x)\right|^{r} e^{r\left|u(x, t)-u_{0}(x)\right|^{2}} d x=0 .
$$

The existence time $T$ can be chosen to satisfy

$$
T^{\frac{\epsilon N}{2}} \rho^{-\epsilon N}+\gamma_{\epsilon} \max \left\{\left\|u_{0}^{r} e^{r u_{0}^{2}}\right\|_{L_{u l, \rho}^{1}\left(\mathbb{R}^{N}\right)}, \rho^{N}\right\}\left(T^{r-\frac{N}{2}(1-\epsilon)} \rho^{-\epsilon N}+T^{r-\frac{N}{2}}\right) \geq \gamma
$$

for any sufficiently small $\epsilon>0$, where $\gamma$ depends only on $N$ and $r$, and $\gamma_{\epsilon}$ is a positive constant depending only on $N, r$ and $\epsilon$ and satisfies $\gamma_{\epsilon} \rightarrow \infty$ as $\epsilon \rightarrow 0$.

(ii) (Critical case) Let $u_{0}$ be a nonnegative measurable function satisfying $\left|u_{0}\right|^{\frac{N}{2}} e^{\frac{N}{2}\left|u_{0}\right|^{2}} \in$ $\mathcal{L}_{u l, \rho}^{1}\left(\mathbb{R}^{N}\right)$. Then there exists a local in time classical solution for problem (1.19) satisfying (1.14) and (1.21) with $r=N / 2$.

(iii) (Nonexistence) Let $0<r<N / 2$. Then there exists a nonnegative measurable data $u_{0}$ such that $\left|u_{0}\right|^{r} e^{r\left|u_{0}\right|^{2}} \in L_{u l, \rho}^{1}\left(\mathbb{R}^{N}\right)$ and problem (1.19) can not possess any local in time classical solution u satisfying (1.14).

We sketch the outline of the proof of our main theorems. In order to argue existence of solutions for (1.1) with general nonlinearity $f$, we introduce a generalization of the Cole-Hopf transformation. In the case $A>1$, let $u$ satisfy $\partial_{t} u=\Delta u+f(u)$ and put

$$
v(x, t):=F(u(x, t))^{-(A-1)} \text {. }
$$

Then $v$ satisfies

$$
\partial_{t} v=\Delta v+(A-1) v^{1+\frac{1}{A-1}}+(A-1) F(u)^{-A-1} f(u)^{-2}|\nabla u|^{2}\left[f^{\prime}(u) F(u)-A\right] .
$$


Using the transformation (1.22), we construct a supersolution of problem (1.1), then we obtain a solution for problem (1.1) by a monotone method. See Proposition 2.1. For construction of a supersolution of (1.1), we utilize the solution for a heat equation with power type nonlinearity, that is,

$$
\partial_{t} v=\Delta v+(A-1) v^{\frac{A}{A-1}}, x \in \mathbb{R}^{N}, t>0, \quad v(x, 0)=F\left(u_{0}(x)\right)^{-(A-1)}, x \in \mathbb{R}^{N} .
$$

Define

$$
\bar{u}(x, t):=F^{-1}\left(v(x, t)^{-1 /(A-1)}\right) .
$$

Then $\bar{u}$ satisfies $\partial_{t} \bar{u} \geq \Delta \bar{u}+f(\bar{u})$, provided that $f^{\prime}(\bar{u}) F(\bar{u}) \leq A$. Then, with the help of the cut-off technique, we can construct a supersolution by using (1.22) and the solution of (1.23). Nonexistence of solutions for problem (1.1) is also proved by using (1.22) with the help of the nonexistence results for (1.23). However, the transformation (1.22) is not useful for the case $A=1$ since the case $A=1$ includes exponential nonlinearity $f(u)=e^{u}$, which is essentially different from power type nonlinearity. For the case $A=1$, we use the transformation

$$
v(x, t):=\log F(u(x, t))^{-1},
$$

instead of (1.22). Under this transformation, the existence problem for (1.1) with general $f$ can be reduced to that of a heat equation with exponential nonlinearity.

The rest of this paper is organized as follows: In Section 2, we give some preliminary results. In particular, we recall the existence and nonexistence results for a heat equation with power type nonlinearity and exponential nonlinearity. In Section 3, we consider local in time existence of solutions for problem (1.1) with the aid of (1.22) and (1.24), and prove Theorems 1.1. In Section 4, we discuss nonexistence of solutions for problem (1.1), and prove Theorems 1.2 and 1.3. In Section 5. we apply our main theorems to several examples of nonlinear heat equations.

\section{Preliminaries}

In this section we recall some properties of uniformly local $L^{p}$ spaces and the existence result of solutions for problem (1.1). Furthermore, in Propositions 2.2 2.5, we discuss the existence result of solutions for problem (1.1) with typical examples of $f$. In particular, we discuss the cases $f(u)=u^{p}$ and $f(u)=e^{u}$.

We first recall two lemmas on properties of uniformly local $L^{p}$ spaces. For partial differential equations in the uniformly local Lebesgue spaces, see for example [2], [13] and [16]. Lemma 2.1] gives the smoothing effect of the heat semigroup in $L_{u l, \rho}^{p}\left(\mathbb{R}^{N}\right)$ (see [2, Proposition 2.1] and [16, Corollary 3.1]). In the following, for any set $X$ and maps $a=a(x)$ and $b=b(x)$ from $X$ to $[0, \infty)$, we say

$$
a(x) \lesssim b(x) \text { for all } x \in X,
$$

if there exists a positive constant $C$ such that $a(x) \leq C b(x)$ for all $x \in X$.

Lemma 2.1. Let $1 \leq p \leq q \leq \infty$. Then there holds

$$
\left\|e^{t \Delta} u\right\|_{L_{u l, \rho}^{q}\left(\mathbb{R}^{N}\right)} \lesssim\left(\rho^{-N\left(\frac{1}{p}-\frac{1}{q}\right)}+t^{-\frac{N}{2}\left(\frac{1}{p}-\frac{1}{q}\right)}\right)\|u\|_{L_{u l, \rho}^{p}\left(\mathbb{R}^{N}\right)}
$$

for all $t>0, \rho>0$ and $u \in L_{u l, \rho}^{p}\left(\mathbb{R}^{N}\right)$.

The following lemma gives basic properties of $\mathcal{L}_{u l, \rho}^{p}\left(\mathbb{R}^{N}\right)$. See [16, Proposition 2.2]. 
Lemma 2.2. Let $1 \leq p<\infty$. The following assertions are equivalent:

$$
\begin{aligned}
& \text { (i) } u \in \mathcal{L}_{u l, \rho}^{p}\left(\mathbb{R}^{N}\right) . \\
& \text { (ii) } \lim _{|y| \rightarrow 0}\|u(\cdot+y)-u(\cdot)\|_{L_{u l, \rho}^{p}\left(\mathbb{R}^{N}\right)}=0 . \\
& \text { (iii) } \lim _{t \rightarrow 0}\left\|e^{t \Delta} u-u\right\|_{L_{u l, \rho}^{p}\left(\mathbb{R}^{N}\right)}=0 .
\end{aligned}
$$

Remark 2.1. $u \in \mathcal{L}_{u l, \rho}^{p}\left(\mathbb{R}^{N}\right)$ is equivalent to $|u|^{p} \in \mathcal{L}_{u l, \rho}^{1}\left(\mathbb{R}^{N}\right)$.

It is pointed out in [16] that the characterization (iii) plays an important role to treat the initial value problem in $\mathcal{L}_{u l, \rho}^{p}\left(\mathbb{R}^{N}\right)$ for a nonlinear heat equation.

We next recall one proposition on existence of solutions for problem (1.1). Proposition 2.1 implies that, if there exists a supersolution for (1.1), we can find a solution of (1.1) below the supersolution via monotone methods. See for example [12, [20] and [22].

Proposition 2.1. Let $f \in C^{1}([0, \infty))$ satisfy (1.2). Let $u_{0} \in L_{u l, \rho}^{1}\left(\mathbb{R}^{N}\right)$ be a nonnegative function. Assume that there exists $\bar{u} \in C^{2,1}\left(\mathbb{R}^{N} \times(0, T)\right)$ satisfying

$$
\bar{u}(x, t) \geq\left(e^{t \Delta} u_{0}\right)(x)+\int_{0}^{t}\left[e^{(t-s) \Delta} f(\bar{u}(\cdot, s))\right](x) d s
$$

for almost every $(x, t) \in \mathbb{R}^{N} \times(0, T)$. Then there exists a solution $u \in C^{2,1}\left(\mathbb{R}^{N} \times(0, T)\right)$ of the integral equation

$$
u(x, t)=\left(e^{t \Delta} u_{0}\right)(x)+\int_{0}^{t}\left[e^{(t-s) \Delta} f(u(\cdot, s))\right](x) d s \quad \text { in } \mathbb{R}^{N} \times(0, T),
$$

which satisfies $\partial_{t} u=\Delta u+f(u)$ in $\mathbb{R}^{N} \times(0, T)$. Furthermore, there holds $0 \leq u(x, t) \leq \bar{u}(x, t)$ in $\mathbb{R}^{N} \times(0, T)$.

Proof. For $n \geq 2$, define the function $u_{n}$ by

$$
u_{n}(x, t):=\left(e^{t \Delta} u_{0}\right)(x)+\int_{0}^{t}\left[e^{(t-s) \Delta} f\left(u_{n-1}(s)\right)\right](x) d s,
$$

where $u_{1}:=0$. Since $f=f(u)$ is an increasing function with respect to $u$ by (1.2) and $\bar{u}$ is a supersolution in the sense of (2.1), if $\bar{u} \geq u_{n-1}(x, t)$, then we have

$$
\begin{aligned}
\bar{u}(x, t) & \geq\left(e^{t \Delta} u_{0}\right)(x)+\int_{0}^{t}\left[e^{(t-s) \Delta} f(\bar{u}(s))\right](x) d s \\
& \geq\left(e^{t \Delta} u_{0}\right)(x)+\int_{0}^{t}\left[e^{(t-s) \Delta} f\left(u_{n-1}(s)\right)\right](x) d s=u_{n}(x, t) .
\end{aligned}
$$

Since $\bar{u}(x, t) \geq 0=u_{1}(x, t)$, by induction we have $\bar{u}(x, t) \geq u_{n}(x, t)$ for all $n \in \mathbb{N}$. By the definition of $u_{n}$ with $n=2$ we first obtain

$$
u_{2}(x, t)=\left(e^{t \Delta} u_{0}\right)(x)+\int_{0}^{t}\left[e^{(t-s) \Delta} f\left(u_{1}(s)\right)\right](x) d s \geq 0=u_{1}(x, t) .
$$

Then, since $f=f(u)$ is an increasing function with respect to $u$, we have

$$
u_{3}=e^{t \Delta} u_{0}+\int_{0}^{t} e^{(t-s) \Delta} f\left(u_{2}(s)\right) d s \geq e^{t \Delta} u_{0}+\int_{0}^{t} e^{(t-s) \Delta} f\left(u_{1}(s)\right) d s=u_{2} .
$$

Repeating the above argument, we have

$$
\bar{u}(x, t) \geq u_{n+1}(x, t) \geq u_{n}(x, t) \geq 0 \quad \text { in } \mathbb{R}^{N} \times(0, T)
$$


for all $n \in \mathbb{N}$. Then we can define the limit function $u$ by

$$
u(x, t):=\lim _{n \rightarrow \infty} u_{n}(x, t),
$$

and by the monotone convergence theorem and (1.2) we see that this function $u$ gives a solution to the desired integral equation. By (2.2) and the monotonicity of $u_{n}$ we have $u(x, t) \leq \bar{u}(x, t)$. Then we can apply the standard regularity theory for parabolic equations and obtain $u \in$ $C^{2,1}\left(\mathbb{R}^{N} \times(0, T)\right)$, so $u$ satisfies the equation in the classical sense. Thus we complete the proof of Proposition 2.1.

We give one lemma on sufficient conditions that equation (1.1) and related inequalities can be rewritten by the integral form.

Lemma 2.3. Let $f \in C^{1}([0, \infty))$ satisfy (1.2). Let $T>0$ and $u \in C^{2,1}\left(\mathbb{R}^{N} \times(0, T)\right)$ satisfy

$$
\lim _{t \rightarrow 0}\left\|\int_{0}^{t} e^{(t-s) \Delta} f(u(\cdot, s)) d s\right\|_{L_{u l, \rho}^{1}\left(\mathbb{R}^{N}\right)}=0 .
$$

(i) Assume that $u$ satisfies $\partial_{t} u=\Delta u+f(u)$ in $\mathbb{R}^{N} \times(0, T)$. If $u_{0} \in L_{u l, \rho}^{1}\left(\mathbb{R}^{N}\right)$ and there holds

$$
\lim _{t \rightarrow 0}\left\|u(t)-e^{t \Delta} u_{0}\right\|_{L_{u l, \rho}^{1}\left(\mathbb{R}^{N}\right)}=0
$$

then $u$ satisfies

$$
u(x, t)=\left(e^{t \Delta} u_{0}\right)(x)+\int_{0}^{t}\left[e^{(t-s) \Delta} f(u(\cdot, s))\right](x) d s \quad \text { in } \mathbb{R}^{N} \times(0, T) .
$$

(ii) Assume that $u$ satisfies $\partial_{t} u \geq \Delta u+f(u)$ in $\mathbb{R}^{N} \times(0, T)$. If $u_{0} \in L_{u l, \rho}^{1}\left(\mathbb{R}^{N}\right)$ and there holds either (2.4) or

$$
u(x, t) \geq\left(e^{t \Delta} u_{0}\right)(x) \quad \text { in } \mathbb{R}^{N} \times(0, T),
$$

then $u$ satisfies

$$
u(x, t) \geq\left(e^{t \Delta} u_{0}\right)(x)+\int_{0}^{t}\left[e^{(t-s) \Delta} f(u(\cdot, s))\right](x) d s \quad \text { in } \mathbb{R}^{N} \times(0, T) .
$$

(iii) Assume that $u$ satisfies $\partial_{t} u \leq \Delta u+f(u)$ in $\mathbb{R}^{N} \times(0, T)$. If $u_{0} \in L_{u l, \rho}^{1}\left(\mathbb{R}^{N}\right)$ and there holds either (2.4) or that there exists a function $r \in C\left([0, T) ; L_{u l, \rho}^{1}\left(\mathbb{R}^{N}\right)\right)$ such that

$$
\|r(\cdot, t)\|_{L_{u l, \rho}^{1}\left(\mathbb{R}^{N}\right)} \rightarrow 0 \quad \text { as } t \rightarrow 0
$$

and

$$
u(x, t) \leq\left(e^{t \Delta} u_{0}\right)(x)+r(x, t) \quad \text { in } \mathbb{R}^{N} \times(0, T),
$$

then $u$ satisfies

$$
u(x, t) \leq\left(e^{t \Delta} u_{0}\right)(x)+\int_{0}^{t}\left[e^{(t-s) \Delta} f(u(\cdot, s))\right](x) d s \quad \text { in } \mathbb{R}^{N} \times(0, T) .
$$

Proof. We first prove assertion (i). Since $u$ satisfies $\partial_{t} u=\Delta u+f(u)$ in $\mathbb{R}^{N} \times(0, T)$, for any sufficiently small $\tau>0$, we have

$$
u(x, t)=\left(e^{(t-\tau) \Delta} u(\tau)\right)(x)+\int_{\tau}^{t}\left[e^{(t-s) \Delta} f(u(s))\right](x) d s \text { in } \mathbb{R}^{N} \times(\tau, T) .
$$


Since $e^{(t-\tau) \Delta} u(\tau)=e^{(t-\tau) \Delta}\left(u(\tau)-e^{\tau \Delta} u_{0}\right)+e^{t \Delta} u_{0}$, by Lemma 2.1 and (2.4) we have

$$
\begin{aligned}
\lim _{\tau \rightarrow 0} & \left\|e^{(t-\tau) \Delta} u(\tau)-e^{t \Delta} u_{0}\right\|_{L^{\infty}\left(\mathbb{R}^{N}\right)} \\
& \lesssim \lim _{\tau \rightarrow 0}\left(\rho^{-N}+(t-\tau)^{-\frac{N}{2}}\right)\left\|u(\tau)-e^{\tau \Delta} u_{0}\right\|_{L_{u l, \rho}^{1}\left(\mathbb{R}^{N}\right)}=0 .
\end{aligned}
$$

On the other hand, since

$$
\int_{0}^{t} e^{(t-s) \Delta} f(u(s)) d s-\int_{\tau}^{t} e^{(t-s) \Delta} f(u(s)) d s=e^{(t-\tau) \Delta} \int_{0}^{\tau} e^{(\tau-s) \Delta} f(u(s)) d s,
$$

by Lemma 2.1 and (2.3) we obtain

$$
\begin{aligned}
\lim _{\tau \rightarrow 0} & \left\|\int_{0}^{t} e^{(t-s) \Delta} f(u(s)) d s-\int_{\tau}^{t} e^{(t-s) \Delta} f(u(s)) d s\right\|_{L^{\infty}\left(\mathbb{R}^{N}\right)} \\
& \lesssim \lim _{\tau \rightarrow 0}\left(\rho^{-N}+(t-\tau)^{-\frac{N}{2}}\right)\left\|\int_{0}^{\tau} e^{(\tau-s) \Delta} f(u(s)) d s\right\|_{L_{u l, \rho}^{1}\left(\mathbb{R}^{N}\right)}=0 .
\end{aligned}
$$

This together with (2.7) and (2.8) proves assertion (i).

Next we prove assertion (ii). If $u_{0}$ satisfies (2.4), then we can prove assertion (ii) as in the above argument. If (2.5) is satisfied, we have

$$
u(x, t) \geq e^{(t-\tau) \Delta} u(\tau)+\int_{\tau}^{t} e^{(t-s) \Delta} f(u(s)) d s \geq e^{t \Delta} u_{0}+\int_{\tau}^{t} e^{(t-s) \Delta} f(u(s)) d s
$$

in $\mathbb{R}^{N} \times(\tau, T)$, where $\tau>0$ is sufficiently small. Then, as in the above argument, we have the convergence of the Duhamel term and assertion (ii) is proved.

Finally, we prove assertion (iii). We only consider the case (2.6). Assuming (2.6), we have

$$
u(t) \leq e^{(t-\tau) \Delta} u(\tau)+\int_{\tau}^{t} e^{(t-s) \Delta} f(u(s)) d s \leq e^{t \Delta} u_{0}+e^{(t-\tau) \Delta} r(\tau)+\int_{\tau}^{t} e^{(t-s) \Delta} f(u(s)) d s
$$

in $\mathbb{R}^{N} \times(\tau, T)$, where $\tau>0$ is sufficiently small. Since $\|r(t)\|_{L_{u l, \rho}^{1}\left(\mathbb{R}^{N}\right)} \rightarrow 0$ as $t \rightarrow 0$, by Lemma 2.1 we have

$$
\left\|e^{(t-\tau) \Delta} r(\tau)\right\|_{L^{\infty}\left(\mathbb{R}^{N}\right)} \lesssim\left(\rho^{-N}+(t-\tau)^{-\frac{N}{2}}\right)\|r(\tau)\|_{L_{u l, \rho}^{1}\left(\mathbb{R}^{N}\right)} \rightarrow 0
$$

as $\tau \rightarrow 0$. Since the convergence of the Duhamel term can be proved as in the above argument, we can prove assertion (iii). Thus we complete the proof of Lemma 2.3.

Now we recall the existence result for the heat equation with power type nonlinearity

$$
\begin{cases}\partial_{t} u=\Delta u+|u|^{p-1} u, & x \in \mathbb{R}^{N}, t>0, \\ u(x, 0)=u_{0}(x), & x \in \mathbb{R}^{N},\end{cases}
$$

where $p>1$. In particular, we consider the case $u_{0}$ belongs to a uniformly local $L^{r}$ space and study local in time existence of solutions for problem (2.9) in suitable functional spaces. We state the existence results for the subcritical case and the critical case, respectively. For existence of classical solutions of (2.9), we study the integral equation

$$
u(t)=e^{t \Delta} u_{0}+\int_{0}^{t} e^{(t-s) \Delta}|u(s)|^{p-1} u(s) d s
$$

in the uniformly local $L^{r}$ spaces. 
Proposition 2.2 (Subcritical case). Let $N \geq 1$ and $p>1$. Assume $r \geq 1$ and $r>\frac{N}{2}(p-$ 1). Given any $u_{0} \in L_{u l, \rho}^{r}\left(\mathbb{R}^{N}\right)$, there exist $T>0$ and at least one classical solution $u \in$ $C\left((0, T) ; L_{\text {ul, },}^{r}\left(\mathbb{R}^{N}\right)\right) \cap L_{l o c}^{\infty}\left((0, T) ; L^{\infty}\left(\mathbb{R}^{N}\right)\right) \cap C^{2,1}\left(\mathbb{R}^{N} \times(0, T)\right)$ satisfying (2.10) and

$$
\lim _{t \rightarrow 0}\left\|u(t)-e^{t \Delta} u_{0}\right\|_{L_{u l, \rho}^{r}\left(\mathbb{R}^{N}\right)}=0 .
$$

The solution $u$ satisfies $\left\|u(t)-u_{0}\right\|_{L_{u l, \rho}^{r}\left(\mathbb{R}^{N}\right)} \rightarrow 0$ as $t \rightarrow 0$, provided that $u_{0} \in \mathcal{L}_{u l, \rho}^{r}\left(\mathbb{R}^{N}\right)$. Furthermore, the maximal existence time $T$ can be estimated to satisfy

$$
T^{\frac{N}{2 p}} \rho^{-\frac{N}{p}}+\left\|u_{0}\right\|_{L_{u l, \rho}^{r}\left(\mathbb{R}^{N}\right)}^{r}\left(T^{\frac{r}{p-1}-\frac{N}{2} \cdot \frac{p-1}{p}} \rho^{-\frac{N}{p}}+T^{\frac{r}{p-1}-\frac{N}{2}}\right) \geq \gamma,
$$

where $\gamma$ is a positive constant depending only on $N, p$ and $r$.

Proposition 2.3 (Critical case). Let $N \geq 1$ and assume $r=\frac{N}{2}(p-1)>1$. Given any $u_{0} \in \mathcal{L}_{u l, \rho}^{r}\left(\mathbb{R}^{N}\right)$, there exist $T>0$ and at least one classical solution $u \in C\left([0, T) ; \mathcal{L}_{u l, \rho}^{r}\left(\mathbb{R}^{N}\right)\right) \cap$ $L_{\text {loc }}^{\infty}\left((0, T) ; L^{\infty}\left(\mathbb{R}^{N}\right)\right) \cap C^{2,1}\left(\mathbb{R}^{N} \times(0, T)\right)$ satisfying (2.10).

One can prove Propositions 2.2 and 2.3 by applying the arguments in [7] and [26] with a slight modification. See Appendix $\mathrm{A}$.

Remark 2.2. (i) Under the assumptions of Proposition 2.2, there exist constants $M>0$ and $T_{0} \in(0, T)$ such that

$$
t^{\sigma}\|u(t)\|_{L_{u l, \rho}^{p r}\left(\mathbb{R}^{N}\right)} \leq M
$$

for all $t \in\left(0, T_{0}\right)$, where $\sigma=\frac{N}{2}\left(\frac{1}{r}-\frac{1}{p r}\right)$. See the proof of Proposition 2.2 in Appendix $A$.

(ii) Under the assumptions of Proposition 2.3, it holds

$$
\lim _{t \rightarrow 0} t^{\sigma}\|u(t)\|_{L_{u l, \rho}^{q}\left(\mathbb{R}^{N}\right)}=0,
$$

where $\max \{p, r\}<q<p r$ and $\sigma=\frac{N}{2}\left(\frac{1}{r}-\frac{1}{q}\right)$. See the proof of Proposition 2.3 in Appendix $A$.

We next discuss existence of solutions for a heat equation with exponential nonlinearity

$$
\begin{cases}\partial_{t} u=\Delta u+e^{u}, & x \in \mathbb{R}^{N}, t>0, \\ u(x, 0)=u_{0}(x), & x \in \mathbb{R}^{N},\end{cases}
$$

where $u_{0}$ satisfies

$$
u_{0}(x) \geq-C e^{|x|^{2-\epsilon}} \quad \text { in } \mathbb{R}^{N}
$$

for some $C>0$ and $\epsilon \in(0,1)$. The problem on existence and nonexistence of solutions for (1.1) with $A=1$ can be reduced to that of (2.12) via the transformation (1.24) under the condition (1.9) or (1.18).

We first prepare one basic lemma on the relationship between the heat semigroup and convex and concave functions. Lemma 2.4 directly follows from the Jensen inequality. For the proof of Lemma 2.4, we refer to [26, Lemma 5.1].

Lemma 2.4. Let $\phi \in L_{u l, \rho}^{1}\left(\mathbb{R}^{N}\right)$ and $J$ be a function from $[0, \infty)$ to itself. If $J$ is convex, then there holds

$$
J\left(e^{t \Delta} \phi(x)\right) \leq\left[e^{t \Delta} J(\phi)\right](x) \text { in } \mathbb{R}^{N} \times(0, \infty) .
$$

On the other hand, if $J$ is concave, then there holds

$$
J\left(e^{t \Delta} \phi(x)\right) \geq\left[e^{t \Delta} J(\phi)\right](x) \text { in } \mathbb{R}^{N} \times(0, \infty) .
$$


Proposition 2.4. Let $N \geq 1$ and $r \geq N / 2$. For any (possibly sign changing) initial data $u_{0}$ satisfying (2.13) and

$$
e^{r u_{0}} \in L_{u l, \rho}^{1}\left(\mathbb{R}^{N}\right) \quad \text { if } r>\frac{N}{2}, \quad e^{r u_{0}} \in \mathcal{L}_{u l, \rho}^{1}\left(\mathbb{R}^{N}\right) \quad \text { if } r=\frac{N}{2},
$$

there exists at least one classical solution $u$ of (2.12) satisfying

$$
u(t)=e^{t \Delta} u_{0}+\int_{0}^{t} e^{(t-s) \Delta} e^{u(s)} d s \quad \text { and } \quad \lim _{t \rightarrow 0}\left\|u(t)-e^{t \Delta} u_{0}\right\|_{L^{\infty}\left(\mathbb{R}^{N}\right)}=0 .
$$

If $r>\frac{N}{2}$, then the existence time $T$ can be taken to satisfy

$$
T^{\frac{\epsilon N}{2}} \rho^{-\epsilon N}+\gamma_{\epsilon}\left\|e^{r u_{0}}\right\|_{L_{u l, \rho}^{1}\left(\mathbb{R}^{N}\right)}\left(T^{r-\frac{N}{2}(1-\epsilon)} \rho^{-\epsilon N}+T^{r-\frac{N}{2}}\right) \geq \gamma
$$

for any sufficiently small $\epsilon>0$, where $\gamma$ depends only on $N$ and $r$, and $\gamma_{\epsilon}$ is a positive constant depending only on $N, r$ and $\epsilon$ satisfying $\gamma_{\epsilon} \rightarrow \infty$ as $\epsilon \rightarrow 0$.

Proof. Let $\epsilon \in(0,1 / 3)$ and $v_{0}:=e^{\frac{\epsilon}{1-\epsilon} u_{0}}$. Put $X_{r}:=L_{u l, \rho}^{\frac{(1-\epsilon) r}{\epsilon}}\left(\mathbb{R}^{N}\right)$ if $r>N / 2$ and $X_{r}:=$ $\mathcal{L}_{u l, \rho}^{\frac{(1-\epsilon) r}{\epsilon}}\left(\mathbb{R}^{N}\right)$ if $r=N / 2$. By the assumption on $u_{0}$ we have $v_{0} \in X_{r}$ for $r \geq N / 2$. Consider

$$
\partial_{t} v=\Delta v+\frac{\epsilon}{1-\epsilon} v^{1 / \epsilon}, \quad x \in \mathbb{R}^{N}, t>0, \quad v(x, 0)=v_{0}(x), \quad x \in \mathbb{R}^{N} .
$$

Since $\frac{(1-\epsilon) r}{\epsilon} \geq \frac{1-\epsilon}{\epsilon} \cdot \frac{N}{2}=\frac{N}{2}\left(\frac{1}{\epsilon}-1\right)>1$, Propositions 2.2 and 2.3 imply existence of a classical nonnegative solution $v$ of (2.15) in $C\left((0, T] ; X_{r}\right)$ if $r>N / 2$ and in $C\left([0, T] ; X_{r}\right)$ if $r=N / 2$, for some $T>0$. Now we apply the Cole-Hopf transformation

$$
\bar{u}(x, t):=\frac{1-\epsilon}{\epsilon} \log v(x, t)
$$

Then a simple calculation shows that $v^{\frac{1-\epsilon}{\epsilon}}=e^{\bar{u}}$ and

$$
\partial_{t} \bar{u}-\Delta \bar{u}=\frac{1-\epsilon}{\epsilon}\left(\frac{\partial_{t} v-\Delta v}{v}+\frac{|\nabla v|^{2}}{v^{2}}\right) \geq e^{\bar{u}} \quad \text { in } \mathbb{R}^{N} \times(0, T) .
$$

Therefore $\bar{u}$ is a supersolution of (2.12). In order to rewrite (2.16) by the integral form, we check the assumptions of Lemma 2.3 (ii). By (2.10) we have $v(t) \geq e^{t \Delta} v_{0}$. Since $\log s$ is monotonically increasing and concave with respect to $s$, by Lemma 2.4 we have

$$
\bar{u}(t)-e^{t \Delta} \bar{u}(0)=\frac{1-\epsilon}{\epsilon}\left(\log v(t)-e^{t \Delta}\left(\log v_{0}\right)\right) \geq \frac{1-\epsilon}{\epsilon}\left(\log v(t)-\log \left(e^{t \Delta} v_{0}\right)\right) \geq 0,
$$

thus $\bar{u}$ satisfies condition (2.5). We now check that $\bar{u}$ satisfies condition (2.3) with $f(u)=e^{u}$. By the definition of $\bar{u}$, it suffices to prove

$$
\int_{0}^{t}\left\|e^{(t-s) \Delta} v(s)^{\frac{1-\epsilon}{\epsilon}}\right\|_{L^{\infty}\left(\mathbb{R}^{N}\right)} d s \rightarrow 0 \text { as } t \rightarrow 0 .
$$

\section{Case $r>N / 2$}

We remark that $v_{0} \in L_{u l, \rho}^{\frac{1-\epsilon}{\epsilon} r}\left(\mathbb{R}^{N}\right)$. If $r>N / 2$, then there exist constants $M>0$ and $T_{0} \in(0, T)$ such that $t^{\sigma}\|v(t)\|_{\substack{L_{u l, \rho}^{\frac{1-\epsilon}{\epsilon^{2}}}\left(\mathbb{R}^{N}\right) \\ \text { s. }}} \leq M$ for $0<t<T_{0}$, where $\sigma=\frac{N}{2}\left(\frac{1}{\frac{1-\epsilon}{\epsilon} r}-\frac{1}{\frac{1-\epsilon}{\epsilon^{2}} r}\right)=\frac{\epsilon N}{2 r}$. See Remark 2.2 (i). Thus, by Lemma 2.1 we have

$$
\begin{aligned}
\int_{0}^{t}\left\|e^{(t-s) \Delta} v(s)^{\frac{1-\epsilon}{\epsilon}}\right\|_{L^{\infty}\left(\mathbb{R}^{N}\right)} d s & \lesssim \int_{0}^{t}\left(\rho^{-2 \sigma}+(t-s)^{-\sigma}\right)\|v(s)\|_{\substack{\frac{1-\epsilon}{\epsilon} \\
L_{u l, \rho}^{\frac{1-\epsilon}{\epsilon^{2}} r}\left(\mathbb{R}^{N}\right)}} d s \\
& \leq \int_{0}^{t}\left(\rho^{-2 \sigma}+(t-s)^{-\sigma}\right) s^{-\frac{1-\epsilon}{\epsilon} \sigma} d s \cdot M^{\frac{1-\epsilon}{\epsilon}} \\
& \lesssim\left(t^{1-\frac{1-\epsilon}{\epsilon} \sigma} \rho^{-2 \sigma}+t^{1-\frac{1-\epsilon}{\epsilon} \sigma-\sigma}\right) M^{\frac{1-\epsilon}{\epsilon}} \rightarrow 0
\end{aligned}
$$


as $t \rightarrow 0$, since $\sigma<\frac{1-\epsilon}{\epsilon} \sigma=\frac{(1-\epsilon) N}{2 r}=\frac{N}{2 r}-\sigma<1$ and $1-\frac{1-\epsilon}{\epsilon} \sigma-\sigma=1-\frac{\sigma}{\epsilon}=1-\frac{N}{2 r}>0$.

Case $r=N / 2$

In the case $r=N / 2$, by Remark 2.2 (ii) we have $t^{\sigma}\|v(t)\|_{L_{u l, \rho}^{\alpha}\left(\mathbb{R}^{N}\right)} \rightarrow 0$ as $t \rightarrow 0$, where $\max \left\{\frac{1-\epsilon}{\epsilon} r, \frac{1}{\epsilon}\right\}<\alpha<\frac{1-\epsilon}{\epsilon^{2}} r$ and $\sigma=\frac{N}{2}\left(\frac{\epsilon}{1-\epsilon} \cdot \frac{1}{r}-\frac{1}{\alpha}\right)$. Therefore, by Lemma 2.1 we have

$$
\begin{aligned}
& \int_{0}^{t}\left\|e^{(t-s) \Delta} v(s)^{\frac{1-\epsilon}{\epsilon}}\right\|_{L^{\infty}\left(\mathbb{R}^{N}\right)} d s \\
& \quad \lesssim \int_{0}^{t}\left(\rho^{-\frac{1-\epsilon}{\epsilon} \cdot \frac{N}{\alpha}}+(t-s)^{-\frac{1-\epsilon}{\epsilon} \cdot \frac{N}{2 \alpha}}\right) s^{-\frac{1-\epsilon}{\epsilon} \cdot \sigma} d s \cdot \sup _{0<s<t} s^{\frac{1-\epsilon}{\epsilon} \sigma}\|v(s)\|_{L_{u l, \rho}^{\frac{1-\epsilon}{\epsilon}}}^{\left.\frac{1}{0} \mathbb{R}^{N}\right)} \\
& \quad \lesssim\left(t^{1-\frac{1-\epsilon}{\epsilon} \sigma} \rho^{-\frac{1-\epsilon}{\epsilon} \cdot \frac{N}{\alpha}}+1\right) \cdot \sup _{0<s<t} s^{\frac{1-\epsilon}{\epsilon} \sigma}\|v(s)\|_{L_{u l, \rho}^{\frac{1-\epsilon}{\epsilon}}}^{\frac{1}{\alpha}}\left(\mathbb{R}^{N}\right) \rightarrow 0
\end{aligned}
$$

as $t \rightarrow 0$, since $-\frac{1-\epsilon}{\epsilon} \cdot \frac{N}{2 \alpha}>-1,-\frac{1-\epsilon}{\epsilon} \sigma>-1$ and $-\frac{1-\epsilon}{\epsilon} \cdot \frac{N}{2 \alpha}-\frac{1-\epsilon}{\epsilon} \sigma+1=0$.

Hence (2.16) can be written by the integral form by Lemma 2.3 (ii) with the aid of (2.17) and (2.18). Then, by Proposition 2.1 we obtain a classical solution $u$ of

$$
u(x, t)=\left(e^{t \Delta} u_{0}\right)(x)+\int_{0}^{t}\left[e^{(t-s) \Delta} e^{u(s)}\right](x) d s \quad \text { in } \mathbb{R}^{N} \times(0, T),
$$

satisfying $u(x, t) \leq \bar{u}(x, t)$. We now prove the convergence of a solution to the initial data. Since $u \leq \bar{u}$, by (2.21) we have

$$
\left\|u(t)-e^{t \Delta} u_{0}\right\|_{L^{\infty}\left(\mathbb{R}^{N}\right)} \leq\left\|\int_{0}^{t} e^{(t-s) \Delta} e^{\bar{u}(s)} d s\right\|_{L^{\infty}\left(\mathbb{R}^{N}\right)} \leq \int_{0}^{t}\left\|e^{(t-s) \Delta} v(s)^{\frac{1-\epsilon}{\epsilon}}\right\|_{L^{\infty}\left(\mathbb{R}^{N}\right)} d s,
$$

and obtain the convergence of $u$ to the initial data by (2.19) and (2.20).

We finally study the estimate of the existence time $T$ for the case $r>N / 2$, and prove (2.14). Let $r>N / 2$ and $\epsilon \in(0,1 / 3)$. One can apply the same argument as in Appendix $\mathrm{A}$ with $p, r$, $\alpha, u^{p}$ replaced by $1 / \epsilon, \frac{1-\epsilon}{\epsilon} r, \frac{\epsilon N}{2 r}, \frac{\epsilon}{1-\epsilon} v^{1 / \epsilon}$, respectively. Then, being careful with the changes of the constants, we see that

$$
T^{r-\frac{N}{2}+\epsilon \frac{N}{2}} \rho^{-\epsilon N}+T^{r-\frac{N}{2}} \geq \frac{\gamma \epsilon^{2 r}}{\left\|v_{0}\right\|_{L_{u l, \rho}^{\frac{1-\epsilon}{\epsilon}}\left(\frac{1-\epsilon}{\epsilon} r\right.}^{L^{\frac{1}{\epsilon}}}\left(\mathbb{R}^{N}\right)},
$$

which proves (2.14) with the help of the definition of $v_{0}$. Thus we complete the proof of Proposition 2.4.

The following result states nonexistence of solutions for exponential nonlinear heat equation including problem (2.12), which shows the optimality of the condition for the integrability of the initial data in Proposition 2.4. Proposition 2.5 is also available for rapidly increasing nonlinearity such as $f(u)=e^{u^{2}}$, and is the key assertion for nonexistence of solutions for problem (1.1) even if (1.18) is violated.

Proposition 2.5. Let $r \in(0, N / 2)$. Let $g$ be a convex function in $\left(s_{0}, \infty\right)$ for some $s_{0}>0$. Assume that $g$ satisfies $g(s) \rightarrow \infty$ as $s \rightarrow \infty, g^{\prime}(s)>0$ for all $s>0$ and

$$
\lim _{s \rightarrow \infty} \frac{g^{\prime \prime}(s)}{\left(g^{\prime}(s)\right)^{2}}=0 .
$$

Then there exists $u_{0} \geq 0$ satisfying

$$
G\left(u_{0}\right)^{-r} \in L_{u l, \rho}^{1}\left(\mathbb{R}^{N}\right) \quad \text { with } \quad G(s):=\int_{s}^{\infty} \frac{d u}{e^{g(u)}}
$$


such that, for every $T>0$, there is no nonnegative solution $u \in C^{2,1}\left(\mathbb{R}^{N} \times(0, T)\right)$ of

$$
u(x, t)=e^{t \Delta} u_{0}+\int_{0}^{t} e^{(t-s) \Delta} e^{g(u(s))} d s \quad \text { in } \mathbb{R}^{N} \times(0, T) .
$$

In particular, there is no nonnegative classical solution $u \in C^{2,1}\left(\mathbb{R}^{N} \times(0, T)\right)$ of

$$
\partial_{t} u=\Delta u+e^{g(u)} \quad \text { in } \mathbb{R}^{N} \times(0, T), \quad u(x, 0)=u_{0}(x) \quad \text { in } \mathbb{R}^{N},
$$

satisfying $\lim _{t \rightarrow 0}\left\|u(t)-e^{t \Delta} u_{0}\right\|_{L^{\infty}\left(\mathbb{R}^{N}\right)}=0$.

For the proof of Proposition 2.5, we introduce one lemma.

Lemma 2.5. Let $g$ be a convex function in $\left(s_{0}, \infty\right)$ such that $g(s) \geq C$ f for all $s \geq s_{0}$ with some $C>0$ and $s_{0}>0$. Let $u_{0} \in L_{u l, \rho}^{1}\left(\mathbb{R}^{N}\right)$ be such that $u_{0}(x) \geq s_{0}$ in $\mathbb{R}^{N}$. Assume that there exists a nonnegative solution $u \in C^{2,1}\left(\mathbb{R}^{N} \times(0, T)\right)$ satisfying

$$
u(x, t)=e^{t \Delta} u_{0}+\int_{0}^{t} e^{(t-s) \Delta} e^{g(u(s))} d s \quad \text { in } \mathbb{R}^{N} \times(0, T) .
$$

Then, for any $k \in \mathbb{N}$ with $k \geq 2$, there exists a constant $C_{k}>0$ such that

$$
\left\|e^{t \Delta} u_{0}\right\|_{L^{\infty}\left(\mathbb{R}^{N}\right)} \leq g^{-1}\left(\frac{k}{k-1} \log \frac{1}{t}+C_{k}\right) \quad \text { for all } t \in(0, T) .
$$

Proof. The proof of Lemma 2.5 relies on the iteration argument developed by Weissler in 26]. Let $k \in \mathbb{N}$ and $k \geq 2$. We first prove by induction that

$$
u(x, t) \geq \frac{t^{a_{l}} C^{\frac{k^{l}-1}{k-1} k} \exp \left(k^{l} \cdot g\left(e^{t \Delta} u_{0}\right)\right)}{\prod_{i=1}^{l}\left(k ! a_{i}\right)^{k^{l-i}}}
$$

for all $l \in \mathbb{N}, x \in \mathbb{R}^{N}$ and $t \in(0, T)$, where $\left\{a_{l}\right\}$ is a sequence defined by $a_{l+1}=k a_{l}+1$ with $a_{1}=k+1$.

We start with the proof for the case $l=1$. Since $u$ satisfies the integral equation, we have $u(x, t) \geq e^{t \Delta} u_{0}(x) \geq s_{0}$ in $\mathbb{R}^{N} \times(0, T)$. Furthermore, since $u(x, t) \geq 0$ satisfies the integral equation and $\eta \in \mathbb{R} \mapsto e^{g(\eta)} \in \mathbb{R}$ is convex, by Lemma 2.4 we have

$$
u(x, t) \geq \int_{0}^{t} e^{(t-s) \Delta} \exp \left(g\left(e^{s \Delta} u_{0}\right)\right) d s \geq \int_{0}^{t} \exp \left(g\left(e^{(t-s) \Delta} e^{s \Delta} u_{0}\right)\right) d s=t e^{g\left(e^{t \Delta} u_{0}\right)} .
$$

It follows from $e^{g(u)} \geq \frac{g(u)^{k}}{k !} \geq \frac{C^{k} u^{k}}{k !}$ for $u \geq s_{0}$, the convexity of $\eta \in \mathbb{R} \mapsto e^{k g(\eta)} \in \mathbb{R}$ and Lemma 2.4 that

$$
\begin{aligned}
u(x, t) & \geq \int_{0}^{t} e^{(t-s) \Delta}\left(\frac{C^{k} u(s)^{k}}{k !}\right) d s \geq \frac{C^{k}}{k !} \int_{0}^{t} e^{(t-s) \Delta}\left(s^{k} e^{k g\left(e^{s \Delta} u_{0}\right)}\right) d s \\
& \geq \frac{C^{k}}{k !} \int_{0}^{t} s^{k} e^{k g\left(e^{t \Delta} u_{0}\right)} d s=\frac{C^{k}}{k !(k+1)} t^{k+1} e^{k g\left(e^{t \Delta} u_{0}\right)} .
\end{aligned}
$$

This proves the inequality (2.24) for the case $l=1$.

Now we assume that (2.24) holds for $l \in \mathbb{N}$. Applying $e^{g(u)} \geq \frac{C^{k} u^{k}}{k !}$ and (2.24) with $l$, we have

$$
u(x, t) \geq \frac{C^{k}}{k !} \int_{0}^{t} e^{(t-s) \Delta}\left(\frac{s^{k a_{l}} C^{\frac{k^{l}-1}{k-1} k^{2}} \exp \left(k^{l+1} g\left(e^{s \Delta} u_{0}\right)\right)}{\prod_{i=1}^{l}\left(k ! a_{i}\right)^{k^{l-i+1}}}\right) d s .
$$


Again, by Lemma 2.4 for the function $\eta \mapsto e^{k^{l+1} g(\eta)}$ we have

$$
\begin{aligned}
u(x, t) & \geq \frac{C^{\frac{k^{l+1}-1}{k-1} k}}{k !} \int_{0}^{t} \frac{s^{k a_{l}} \exp \left(k^{l+1} g\left(e^{t \Delta} u_{0}\right)\right)}{\prod_{i=1}^{l}\left(k ! a_{i}\right)^{k^{l-i+1}}} d s \\
& =\frac{t^{k a_{l}+1} C^{\frac{k^{l+1}-1}{k-1} k} \exp \left(k^{l+1} g\left(e^{t \Delta} u_{0}\right)\right)}{k !\left(k a_{l}+1\right) \prod_{i=1}^{l}\left(k ! a_{i}\right)^{k^{l-i+1}}}=\frac{t^{a_{l+1}} C^{\frac{k^{l+1}-1}{k-1} k} \exp \left(k^{l+1} g\left(e^{t \Delta} u_{0}\right)\right)}{\prod_{i=1}^{l+1}\left(k ! a_{i}\right)^{k^{l-i+1}}} .
\end{aligned}
$$

Here we used the relation $a_{l+1}=k a_{l}+1$. This implies that (2.24) holds with $l+1$. Thus we complete the proof of (2.24).

We now prove Lemma 2.5. It is easy to see that

$$
a_{l}=\frac{k}{k-1} \cdot k^{l}-\frac{1}{k-1}
$$

for all $l \in \mathbb{N}$. Therefore, it follows from (2.24) that

$$
u(x, t)^{\frac{1}{k^{l}}} \prod_{i=1}^{l}\left(k ! a_{i}\right)^{k^{-i}} \geq t^{\frac{k}{k-1}-\frac{1}{k^{l}(k-1)}} C^{\frac{k}{k-1}-\frac{k}{k^{l}(k-1)}} e^{g\left(e^{t \Delta} u_{0}\right)}
$$

for all $l \in \mathbb{N}$. Taking $l \rightarrow \infty$, we have

$$
\prod_{i=1}^{\infty}\left(k ! a_{i}\right)^{k^{-i}} \geq t^{\frac{k}{k-1}} C^{\frac{k}{k-1}} e^{g\left(e^{t \Delta} u_{0}\right)} .
$$

Remark that the left hand side of (2.25) converges. Indeed, we can easily see that

$$
\log \left(\prod_{i=1}^{\infty}\left(k ! a_{i}\right)^{k^{-i}}\right) \leq \sum_{i=1}^{\infty} \frac{(i+1) \log k+\log (k !)}{k^{i}}<\infty .
$$

Then we obtain the assertion of the lemma from (2.25), and complete the proof of Lemma 2.5 ,

Proof of Proposition 2.5. The proof is by contradiction. Fix $r<N / 2$ and $2<\alpha<N / r$. Let $\epsilon>0$ be a sufficiently small constant such that $(1+\epsilon) \alpha r<N$. Since $g^{\prime}>0$ and $g$ is convex, taking a sufficiently large $s_{0}>0$ if necessary, we can take a constant $C>0$ such that $g(s) \geq C s$ for all $s \geq s_{0}$. Define

$$
u_{0}(x):=\left\{\begin{array}{lll}
g^{-1}\left(\alpha \log \frac{1}{|x|}\right) & \text { if } & |x|<r_{0} \\
s_{0} & \text { if } & |x| \geq r_{0}
\end{array}\right.
$$

where $r_{0}>0$ is chosen to satisfy $g^{-1}\left(\alpha \log \left(1 / r_{0}\right)\right)=s_{0}$. Then, by $(1+\epsilon) \alpha r<N$ we have $e^{(1+\epsilon) r g\left(u_{0}\right)} \in L_{u l, \rho}^{1}\left(\mathbb{R}^{N}\right)$. Taking a sufficiently large $s_{0}>0$ if necessary, by (2.22) we may assume that $g^{\prime \prime}(s) \leq \epsilon\left(g^{\prime}(s)\right)^{2}$ for all $s \geq s_{0}$. Then we can easily see that

$$
g^{\prime}(s) \leq g^{\prime}\left(s_{0}\right) e^{\epsilon\left(g(s)-g\left(s_{0}\right)\right)} \leq g^{\prime}\left(s_{0}\right) e^{\epsilon g(s)}
$$

for all $s \geq s_{0}$. On the other hand, since it follows from (2.22) that

$$
\frac{g^{\prime \prime}(s)}{\left(g^{\prime}(s)\right)^{2}} e^{-g(s)} \leq \frac{1}{2} \cdot \frac{g^{\prime \prime}(s)}{\left(g^{\prime}(s)\right)^{2}} e^{-g(s)}+\frac{1}{2} e^{-g(s)}=\frac{1}{2}\left(-\frac{1}{g^{\prime}(s)} e^{-g(s)}\right)^{\prime}
$$


for sufficiently large $s>0$, we have

$$
\int_{s}^{\infty} \frac{g^{\prime \prime}(u)}{\left(g^{\prime}(u)\right)^{2}} e^{-g(u)} d u \leq \frac{1}{2} \cdot \frac{1}{g^{\prime}(s)} e^{-g(s)}
$$

and by (2.26) we obtain

$$
\begin{aligned}
G(s) & =\int_{s}^{\infty} \frac{d u}{e^{g(u)}}=\frac{1}{g^{\prime}(s)} e^{-g(s)}-\int_{s}^{\infty} \frac{g^{\prime \prime}(u)}{\left(g^{\prime}(u)\right)^{2}} e^{-g(u)} d u \\
& \geq \frac{1}{2} \cdot \frac{1}{g^{\prime}(s)} e^{-g(s)} \geq \frac{1}{2} \cdot \frac{1}{g^{\prime}\left(s_{0}\right)} e^{-(1+\epsilon) g(s)}
\end{aligned}
$$

for all sufficiently large $s>0$. This together with $e^{(1+\epsilon) g\left(u_{0}\right)} \in L_{u l, \rho}^{1}\left(\mathbb{R}^{N}\right)$ implies that $G\left(u_{0}\right)^{-r} \in$ $L_{u l, \rho}^{1}\left(\mathbb{R}^{N}\right)$.

Putting $y=\sqrt{t} z$, we have

$$
\begin{aligned}
\left\|e^{t \Delta} u_{0}\right\|_{L^{\infty}\left(\mathbb{R}^{N}\right)} & \geq(4 \pi t)^{-\frac{N}{2}} \int_{|y| \leq r_{0}} e^{-\frac{|y|^{2}}{4 t}} g^{-1}\left(\alpha \log \frac{1}{|y|}\right) d y \\
& =(4 \pi)^{-\frac{N}{2}} \int_{|z| \leq r_{0} t^{-1 / 2}} e^{-\frac{|z|^{2}}{4}} g^{-1}\left(\frac{\alpha}{2} \log \frac{1}{t}+\alpha \log \frac{1}{|z|}\right) d z
\end{aligned}
$$

Let $\delta>0$ be a sufficiently small constant such that $\alpha / 2-\delta>1+2 \delta$. Then we have $t^{-\delta / \alpha} \leq$ $r_{0} t^{-1 / 2}$ for all sufficiently small $t>0$ and

$$
\begin{aligned}
\left\|e^{t \Delta} u_{0}\right\|_{L^{\infty}\left(\mathbb{R}^{N}\right)} & \geq(4 \pi)^{-\frac{N}{2}} \int_{|z| \leq t^{-\delta / \alpha}} e^{-\frac{|z|^{2}}{4}} g^{-1}\left(\frac{\alpha}{2} \log \frac{1}{t}+\alpha \log \frac{1}{|z|}\right) d z \\
& \geq g^{-1}\left(\left(\frac{\alpha}{2}-\delta\right) \log \frac{1}{t}\right) \cdot(4 \pi)^{-\frac{N}{2}} \int_{|z| \leq t^{-\delta / \alpha}} e^{-\frac{|z|^{2}}{4}} d z \\
& =g^{-1}\left(\left(\frac{\alpha}{2}-\delta\right) \log \frac{1}{t}\right) \cdot\left(1+O\left(e^{-\frac{t^{-2 \delta / \alpha}}{8}}\right)\right)
\end{aligned}
$$

for all sufficiently small $t>0$. We prove that this yields a contradiction. Assume that there exists a nonnegative classical solution $u$ of

$$
u(t)=e^{t \Delta} u_{0}+\int_{0}^{t} e^{(t-s) \Delta} e^{g(u(s))} d s .
$$

Then, by Lemma 2.5 and (2.27) we have

$$
g^{-1}\left(\left(\frac{\alpha}{2}-\delta\right) \log \frac{1}{t}\right) \cdot\left(1+O\left(e^{-\frac{t^{-2 \delta / \alpha}}{8}}\right)\right) \leq g^{-1}\left(\left(\frac{k}{k-1}+\delta\right) \log \frac{1}{t}\right)
$$

for all sufficiently small $t>0$. Here we take a sufficiently large $k \in \mathbb{N}$ satisfying $k /(k-1)+\delta<$ $1+2 \delta$. Then, since $\alpha / 2-\delta>1+2 \delta$, we have $k /(k-1)+\delta<\alpha / 2-\delta$. In the following, we prove that (2.28) yields a contradiction. For simplicity, define $a:=\alpha / 2-\delta, b:=k /(k-1)+\delta$, $c:=2 \delta / \alpha$ and $\tau:=\log (1 / t)$. Then $a>b$ and (2.28) implies that

$$
g^{-1}(a \tau) \cdot\left(1+O\left(e^{-e^{-c \tau} / 8}\right)\right) \leq g^{-1}(b \tau)
$$

for sufficiently large $\tau>0$. Since $\left(g^{-1}(s)\right)^{\prime}=1 / g^{\prime}\left(g^{-1}(s)\right)>0$, by the mean value theorem and (2.26) we see that there exists a constant $d>0$ such that

$$
g^{-1}(a \tau) \geq g^{-1}(b \tau)+\frac{1}{g^{\prime}\left(g^{-1}(a \tau)\right)}(a-b) \tau \geq g^{-1}(b \tau)+\frac{d}{e^{\epsilon a \tau}}(a-b) \tau,
$$


which implies that

$$
g^{-1}(a \tau) \cdot\left(1+O\left(e^{-e^{-c \tau} / 8}\right)\right) \geq g^{-1}(b \tau)+\frac{d}{e^{\epsilon a \tau}}(a-b) \tau+O\left(g^{-1}(a \tau) e^{-e^{-c \tau} / 8}\right)>g^{-1}(b \tau)
$$

for all sufficiently large $\tau>0$. Remark that $g^{-1}(a \tau)=O(\tau)$ for all sufficiently large $\tau$ since $g(s) \geq C s$ for all $s \geq s_{0}$. This contradicts (2.29), and so (2.28) yields a contradiction.

We finally prove the latter assertion of Proposition 2.5. Assume that there exist a constant $T>0$ and a nonnegative classical solution $u \in C^{2,1}\left(\mathbb{R}^{N} \times(0, T)\right)$ of (2.23) satisfying $\lim _{t \rightarrow 0} \| u(t)-$ $e^{t \Delta} u_{0} \|_{L^{\infty}\left(\mathbb{R}^{N}\right)}=0$. Then, for any $\tau \in(0, T)$, by (2.23) we have

$$
u(x, t)=\left(e^{(t-\tau) \Delta} u(\tau)\right)(x)+\int_{\tau}^{t}\left[e^{(t-s) \Delta} e^{g(u(s))}\right](x) d s
$$

in $\mathbb{R}^{N} \times(\tau, T)$. This implies that

$$
\int_{\tau}^{t}\left[e^{(t-s) \Delta} e^{g(u(s))}\right](x) d s \leq u(x, t)<\infty,
$$

so we see that

$$
\lim _{\tau \rightarrow 0} \int_{\tau}^{t}\left[e^{(t-s) \Delta} e^{g(u(s))}\right](x) d s=\int_{0}^{t}\left[e^{(t-s) \Delta} e^{g(u(s))}\right](x) d s
$$

exists for all $(x, t) \in \mathbb{R}^{N} \times(0, T)$. Therefore, since $\left\|u(\tau)-e^{\tau \Delta} u_{0}\right\|_{L^{\infty}\left(\mathbb{R}^{N}\right)} \rightarrow 0$ as $\tau \rightarrow 0$, as in the proof of Lemma 2.3, taking the limit $\tau \rightarrow 0$ in (2.30), we see that $u$ satisfies the integral equation

$$
u(x, t)=e^{t \Delta} u_{0}+\int_{0}^{t} e^{(t-s) \Delta} e^{g(u(s))} d s \quad \text { in } \mathbb{R}^{N} \times(0, T) .
$$

This is a contradiction. Thus we complete the proof of Proposition 2.5

\section{Existence of a solution for problem (1.1)}

In this section we show local and global in time existence of solutions for problem (1.1) with the help of Propositions 2.1 2.4, Recall that

$$
A=\lim _{s \rightarrow \infty} f^{\prime}(s) F(s) .
$$

Before starting the proof of main theorems, we prepare two lemmas.

Lemma 3.1. Let $f \in C^{1}([0, \infty))$ satisfy (1.2) and (1.8). Define $g(s):=f\left(F^{-1}(s)\right)$. Assume that (1.9) holds for some $s_{1}>0$. Then

$$
g(s) \lesssim s^{-A}
$$

for all sufficiently small $s>0$.

Proof. It follows from $\left(F^{-1}(s)\right)^{\prime}=-f\left(F^{-1}(s)\right)=-g(s)$ that

$$
g^{\prime}(s)=f^{\prime}\left(F^{-1}(s)\right)\left(F^{-1}(s)\right)^{\prime}=-f^{\prime}\left(F^{-1}(s)\right) g(s) .
$$

Since $F^{-1}$ is monotonically decreasing with respect to $s$ and $F^{-1}(s) \rightarrow \infty$ as $s \rightarrow 0$, we have $F^{-1}(s)>s_{1}$ for all sufficiently small $s>0$. This together with (1.9) implies that

$$
s f^{\prime}\left(F^{-1}(s)\right)=f^{\prime}\left(F^{-1}(s)\right) F\left(F^{-1}(s)\right) \leq A
$$

for all sufficiently small $s>0$. Combining (3.2) and (3.3), we have

$$
g^{\prime}(s) \geq-A s^{-1} g(s)
$$

for all sufficiently small $s>0$. This proves Lemma 3.1 . 
Lemma 3.2. Let $f \in C^{1}([0, \infty))$ satisfy (1.2) and (1.8). Let $A>1$ and $s_{1}>0$.

(i) If $f^{\prime}(s) F(s) \leq A$ for all $s>s_{1}$, then $F(s)^{-(A-1)}$ is convex in $\left(s_{1}, \infty\right)$. On the other hand, if $f^{\prime}(s) F(s) \geq A$ for all $s>s_{1}$, then $F(s)^{-(A-1)}$ is concave in $\left(s_{1}, \infty\right)$.

(ii) If $f^{\prime}(s) F(s) \leq 1$ for all $s>s_{1}$, then $\log F(s)^{-1}$ is convex in $\left(s_{1}, \infty\right)$. On the other hand, if $f^{\prime}(s) F(s) \geq 1$ for all $s>s_{1}$, then $\log F(s)^{-1}$ is concave in $\left(s_{1}, \infty\right)$.

Proof. Assume that $f^{\prime}(s) F(s) \leq A$ for all $s>s_{1}$. Then direct calculations show that

$$
\frac{d^{2}}{d s^{2}}\left(F(s)^{-(A-1)}\right)=(A-1) F(s)^{-A-1} \frac{1}{f(s)^{2}}\left(A-f^{\prime}(s) F(s)\right) \geq 0
$$

for all $s>s_{1}$. This proves the convexity of $F(s)^{-(A-1)}$ in assertion (i). Other cases can be treated in the same manner.

The rest of this section is devoted to the proof of Theorem 1.1.

\section{Proof of Theorem 1.1.}

\section{$\underline{\text { Case } A>1}$}

We first consider the case $A>1$, and prove local in time existence of a solution for (1.1). Let $r$ be a constant given in the assumption of Theorem 1.1. Define

$$
v_{0}(x):=\max \left\{F\left(u_{0}(x)\right)^{-(A-1)}, F\left(s_{1}\right)^{-(A-1)}\right\},
$$

where $s_{1}$ is the constant appearing in (1.9). In particular, we have

$$
v_{0}(x) \geq F\left(s_{1}\right)^{-(A-1)}>0 \quad \text { in } \mathbb{R}^{N} .
$$

Consider the semilinear heat equation

$$
\partial_{t} v=\Delta v+(A-1) v^{\frac{A}{A-1}} \quad \text { in } \mathbb{R}^{N} \times(0, T), \quad v(x, 0)=v_{0}(x) \quad \text { in } \mathbb{R}^{N},
$$

where $T>0$. By (3.4) we have

$$
\sup _{y \in \mathbb{R}^{N}} \int_{B_{\rho}(y)}\left|v_{0}(x)\right|^{\frac{r}{A-1}} d x=\sup _{y \in \mathbb{R}^{N}} \int_{B_{\rho}(y)} \max \left\{F\left(u_{0}(x)\right)^{-r}, F\left(s_{1}\right)^{-r}\right\} d x,
$$

which implies that $v_{0} \in L_{u l, \rho}^{r /(A-1)}\left(\mathbb{R}^{N}\right)$ in case of (1.11) and $v_{0} \in \mathcal{L}_{u l, \rho}^{r /(A-1)}\left(\mathbb{R}^{N}\right)$ in case of (1.17). Since

- $\frac{r}{A-1}>\frac{N}{2(A-1)}=\frac{N}{2}\left[\frac{A}{A-1}-1\right]$ and $\frac{r}{A-1} \geq 1$ if (1.10) holds;

- $\frac{r}{A-1} \geq \frac{N}{2(A-1)}=\frac{N}{2}\left[\frac{A}{A-1}-1\right]$ and $\frac{r}{A-1}>1$ if (1.16) holds,

in view of Propositions 2.2 and 2.3 , there exist a constant $T>0$ and a classical solution $v$ in $L_{u l, \rho}^{r /(A-1)}\left(\mathbb{R}^{N}\right)$ (resp. in $\mathcal{L}_{u l, \rho}^{r /(A-1)}\left(\mathbb{R}^{N}\right)$ ) of (3.6) satisfying

$$
v(x, t)=\left(e^{t \Delta} v_{0}\right)(x)+\int_{0}^{t}\left[e^{(t-s) \Delta} v(s)^{\frac{A}{A-1}}\right](x) d s \quad \text { in } \mathbb{R}^{N} \times(0, T),
$$

if $r$ satisfies (1.10) (resp. $r$ satisfies (1.16)). Then we have $v \in C^{2,1}\left(\mathbb{R}^{N} \times(0, T)\right)$. Furthermore, by (3.5) and (3.8) we obtain

$$
v(x, t) \geq\left(e^{t \Delta} v_{0}\right)(x) \geq F\left(s_{1}\right)^{-(A-1)}
$$


in $\mathbb{R}^{N} \times(0, T)$.

Define the function $\bar{u} \in C^{2,1}\left(\mathbb{R}^{N} \times(0, T)\right)$ by

$$
\bar{u}(x, t):=F^{-1}\left(v(x, t)^{-1 /(A-1)}\right) .
$$

Then, by (3.9) we have

$$
\bar{u}(x, t) \geq F^{-1}\left(F\left(s_{1}\right)\right)=s_{1} \quad \text { in } \mathbb{R}^{N} \times(0, T) .
$$

Since we have

$$
\begin{aligned}
\partial_{t} \bar{u} & =\frac{1}{A-1} f(\bar{u}) v^{-\frac{A}{A-1}} \partial_{t} v, \\
\Delta \bar{u} & =\frac{f^{\prime}(\bar{u}) f(\bar{u}) v^{-\frac{2 A}{A-1}}|\nabla v|^{2}}{(A-1)^{2}}-\frac{A f(\bar{u}) v^{-\frac{A}{A-1}-1}|\nabla v|^{2}}{(A-1)^{2}}+\frac{f(\bar{u}) v^{-\frac{A}{A-1}} \Delta v}{A-1},
\end{aligned}
$$

by (3.6) we obtain

$$
\begin{aligned}
\partial_{t} \bar{u}-\Delta \bar{u}-f(\bar{u}) & =\frac{1}{(A-1)^{2}} f(\bar{u}) v^{-\frac{A}{A-1}-1}|\nabla v|^{2}\left[A-f^{\prime}(\bar{u}) v^{-\frac{1}{A-1}}\right] \\
& =\frac{1}{(A-1)^{2}} f(\bar{u}) v^{-\frac{A}{A-1}-1}|\nabla v|^{2}\left[A-f^{\prime}(\bar{u}) F(\bar{u})\right] .
\end{aligned}
$$

Thus, since $f^{\prime}(\bar{u}) F(\bar{u}) \leq A$ in $\mathbb{R}^{N} \times(0, T)$ by (1.9) and (3.11), we obtain

$$
\partial_{t} \bar{u} \geq \Delta \bar{u}+f(\bar{u}) \quad \text { in } \mathbb{R}^{N} \times(0, T) .
$$

Furthermore, by (3.4) we see that

$$
\bar{u}(x, 0)=\max \left\{u_{0}(x), s_{1}\right\} \geq u_{0}(x) \quad \text { in } \mathbb{R}^{N} .
$$

By (3.10) and (3.11) we obtain $F^{-1}\left(v(x, t)^{-1 /(A-1)}\right) \geq s_{1}$. This together with Lemma 3.1 with $A>1$ implies that

$$
\int_{0}^{t} e^{(t-s) \Delta} f(\bar{u}(s)) d s=\int_{0}^{t} e^{(t-s) \Delta} f\left(F^{-1}\left(v(s)^{-\frac{1}{A-1}}\right)\right) d s \lesssim \int_{0}^{t} e^{(t-s) \Delta} v(s)^{\frac{A}{A-1}} d s
$$

for all $(x, t) \in \mathbb{R}^{N} \times(0, T)$. For the case $r=N / 2$, since $\frac{r}{A-1}=\frac{N}{2}\left(\frac{A}{A-1}-1\right)$ and $v \in$ $C\left([0, T] ; \mathcal{L}_{u l, \rho}^{r /(A-1)}\left(\mathbb{R}^{N}\right)\right)$, we have

$$
\int_{0}^{t} e^{(t-s) \Delta} v(s)^{\frac{A}{A-1}} d s=v(t)-e^{t \Delta} v_{0} \rightarrow 0 \quad \text { in } L_{u l, \rho}^{\frac{r}{A-1}}\left(\mathbb{R}^{N}\right),
$$

as $t \rightarrow 0$. For the case $r>N / 2$, by Proposition 2.2 we also have (3.16). Then, since $\frac{r}{A-1} \geq 1$, by (3.15) and (3.16) we have (2.3) for $\bar{u}$. On the other hand, by Lemma 2.4, Lemma 3.2, (1.9) and (3.14) we have

$$
F\left(e^{t \Delta} \bar{u}(0)\right)^{-(A-1)} \leq e^{t \Delta}\left[F(\bar{u}(0))^{-(A-1)}\right] .
$$

This together with (3.9) and (3.10) implies that

$$
F(\bar{u}(t))^{-(A-1)}-F\left(e^{t \Delta} \bar{u}(0)\right)^{-(A-1)} \geq v(t)-e^{t \Delta}\left[F(\bar{u}(0))^{-(A-1)}\right]=v(t)-e^{t \Delta} v_{0} \geq 0 .
$$

Since $F(s)^{-(A-1)}$ is monotonically increasing with respect to $s$, we see that $\bar{u}(x, t) \geq\left(e^{t \Delta} \bar{u}(0)\right)(x)$ in $\mathbb{R}^{N} \times(0, T)$. Therefore we can apply Lemma 2.3 (ii) and by (3.13) and (3.14) we obtain

$$
\bar{u}(x, t) \geq\left(e^{t \Delta} \bar{u}(0)\right)(x)+\int_{0}^{t}\left[e^{(t-s) \Delta} f(\bar{u}(s))\right](x) d s \geq\left(e^{t \Delta} u_{0}\right)(x)+\int_{0}^{t}\left[e^{(t-s) \Delta} f(\bar{u}(s))\right](x) d s
$$


for all $(x, t) \in \mathbb{R}^{N} \times(0, T)$. Hence we can apply Proposition 2.1 and prove existence of a local in time classical solution $u$ for problem (1.1). Note that, in case of (1.10), the existence time $T$ satisfies

$$
\begin{aligned}
& T^{\frac{N}{2}\left(1-\frac{1}{A}\right)} \rho^{-N\left(1-\frac{1}{A}\right)}+\gamma_{0} \max \left\{\left\|F\left(u_{0}\right)^{-r}\right\|_{L_{u l, \rho}^{1}\left(\mathbb{R}^{N}\right)}, F\left(s_{1}\right)^{-r} \rho^{N}\right\}\left(T^{r-\frac{N}{2 A}} \rho^{-N \cdot \frac{A-1}{A}}+T^{r-\frac{N}{2}}\right) \\
& \geq T^{\frac{N}{2}\left(1-\frac{1}{A}\right)} \rho^{-N\left(1-\frac{1}{A}\right)}+\left\|v_{0}\right\|_{L_{u l, \rho}^{\frac{r}{A-1}}}^{\frac{r}{A-1}\left(\mathbb{R}^{N}\right)} \\
&
\end{aligned}
$$

by (2.11) with $p=\frac{A}{A-1}$, (3.4) and (3.7), where $\gamma_{0}$ and $\gamma_{1}$ are positive constants depending only on $N, A$ and $r$. Thus we obtain (1.13).

It remains to prove (1.12). By Proposition 2.1 we have $u(x, t) \leq \bar{u}(x, t)$. Then, by (1.2) and (3.15) we have

$$
\left|u(t)-e^{t \Delta} u_{0}\right|=\int_{0}^{t} e^{(t-s) \Delta} f(u(s)) d s \leq \int_{0}^{t} e^{(t-s) \Delta} f(\bar{u}(s)) d s \lesssim \int_{0}^{t} e^{(t-s) \Delta} v(s)^{\frac{A}{A-1}} d s .
$$

This together with (3.16) yields (1.12). Thus we complete the proof of Theorem 1.1 for the case $A>1$.

\section{Case $A=1$}

We next consider the case $A=1$. The proof is similar to the above argument. Let

$$
w_{0}(x):=\max \left\{\log F\left(u_{0}(x)\right)^{-1}, \log F\left(s_{1}\right)^{-1}\right\} .
$$

Then we have

$$
w_{0}(x) \geq \log F\left(s_{1}\right)^{-1} \quad \text { in } \mathbb{R}^{N} .
$$

Consider the semilinear heat equation

$$
\partial_{t} w=\Delta w+e^{w} \quad \text { in } \mathbb{R}^{N} \times(0, T), \quad w(x, 0)=w_{0}(x) \quad \text { in } \mathbb{R}^{N},
$$

where $T>0$. Since (1.11) and (3.17) yields

$$
\sup _{y \in \mathbb{R}^{N}} \int_{B_{\rho}(y)} e^{r w_{0}(x)} d x=\sup _{y \in \mathbb{R}^{N}} \int_{B_{\rho}(y)} \max \left\{F\left(u_{0}(x)\right)^{-r}, F\left(s_{1}\right)^{-r}\right\} d x
$$

we have $e^{r w_{0}} \in L_{u l, \rho}^{1}\left(\mathbb{R}^{N}\right)$ if $r>N / 2$ and $e^{r w_{0}} \in \mathcal{L}_{u l, \rho}^{1}\left(\mathbb{R}^{N}\right)$ if $r=N / 2$. Hence, by Proposition 2.4 we can find a constant $T>0$ and a classical solution $w$ of (3.19) satisfying

$$
w(t)=e^{t \Delta} w_{0}+\int_{0}^{t} e^{(t-s) \Delta} e^{w(s)} d s \geq e^{t \Delta} w_{0}
$$

and

$$
\lim _{t \rightarrow 0}\left\|w(t)-e^{t \Delta} w_{0}\right\|_{L^{\infty}\left(\mathbb{R}^{N}\right)}=\lim _{t \rightarrow 0}\left\|\int_{0}^{t} e^{(t-s) \Delta} e^{w(s)} d s\right\|_{L^{\infty}\left(\mathbb{R}^{N}\right)}=0 .
$$

Define the function $\bar{u} \in C^{2,1}\left(\mathbb{R}^{N} \times(0, T)\right)$ by

$$
\bar{u}(x, t):=F^{-1}\left(e^{-w(x, t)}\right) .
$$

Then, as in (3.11), by (3.18) we have

$$
\bar{u}(x, t) \geq s_{1} \quad \text { in } \mathbb{R}^{N} \times(0, T) .
$$


One can easily check that

$$
\partial_{t} \bar{u}=f(\bar{u}) e^{-w} \partial_{t} w, \quad \Delta \bar{u}=f^{\prime}(\bar{u}) f(\bar{u}) e^{-2 w}|\nabla w|^{2}-f(\bar{u}) e^{-w}|\nabla w|^{2}+f(\bar{u}) e^{-w} \Delta w,
$$

and so we have

$$
\partial_{t} \bar{u}-\Delta \bar{u}-f(\bar{u})=f(\bar{u}) e^{-w}|\nabla w|^{2}\left(1-f^{\prime}(\bar{u}) e^{-w}\right)=f(\bar{u}) e^{-w}|\nabla w|^{2}\left(1-f^{\prime}(\bar{u}) F(\bar{u})\right) .
$$

Since $f^{\prime}(\bar{u}) F(\bar{u}) \leq 1$ by (1.9) with $A=1$ and (3.23), we have

$$
\partial_{t} \bar{u} \geq \Delta \bar{u}+f(\bar{u}) \quad \text { in } \mathbb{R}^{N} \times(0, T) .
$$

By the similar argument as in the case $A>1$ with the aid of Lemma 3.2 we obtain $\bar{u}(x, t) \geq$ $\left(e^{t \Delta} \bar{u}(0)\right)(x)$. Furthermore, by Lemma 3.1 with $A=1$ we have

$$
\int_{0}^{t} e^{(t-s) \Delta} f(\bar{u}(s)) d s=\int_{0}^{t} e^{(t-s) \Delta} f\left(F^{-1}\left(e^{-w}\right)\right) d s \lesssim \int_{0}^{t} e^{(t-s) \Delta} e^{w} d s .
$$

Then, by (3.21) and (3.24), we can apply Lemma 2.3 (ii), and by (3.17) we obtain

$$
\bar{u}(x, t) \geq e^{t \Delta} u_{0}+\int_{0}^{t} e^{(t-s) \Delta} f(\bar{u}(s)) d s .
$$

Applying Proposition 2.1, we obtain a local in time classical solution $u$ of problem (1.1). Note that, if $r>\frac{N}{2}$, by (2.14), (3.17) and (3.20) we see that the existence time $T$ can be taken to satisfy

$$
\begin{aligned}
T^{\frac{\epsilon N}{2}} \rho^{-\epsilon N}+C \gamma_{\epsilon} \max \left\{\left\|e^{r u_{0}}\right\|_{L_{u l, \rho}^{1}\left(\mathbb{R}^{N}\right)}, F\left(s_{1}\right)^{-r} \rho^{N}\right\}\left(T^{r-\frac{N}{2}(1-\epsilon)} \rho^{-\epsilon N}+T^{r-\frac{N}{2}}\right) \\
\geq T^{\frac{\epsilon N}{2}} \rho^{-\epsilon N}+\gamma_{\epsilon}\left\|e^{r w_{0}}\right\|_{L_{u l, \rho}^{1}\left(\mathbb{R}^{N}\right)}\left(T^{r-\frac{N}{2}(1-\epsilon)} \rho^{-\epsilon N}+T^{r-\frac{N}{2}}\right) \geq \gamma_{*}
\end{aligned}
$$

for all sufficiently small $\epsilon>0$, where $C>0$ is a constant and $\gamma_{*}$ depends only on $N$ and $r$, and $\gamma_{\epsilon}$ is a constant depending on $N, r$ and $\epsilon$ such that $\gamma_{\epsilon} \rightarrow \infty$ as $\epsilon \rightarrow 0$. Thus we obtain (1.15).

Convergence of the solution $u$ to the initial data (1.14) is proved as follows. Since $u(x, t) \leq$ $\bar{u}(x, t)=F^{-1}\left(e^{-w}\right)$ and $F^{-1}\left(e^{-w}\right) \geq s_{1}$ by (3.22) and (3.23), we can apply Lemma 3.1 with $A=1$ and obtain

$$
\left|u(t)-e^{t \Delta} u_{0}\right|=\int_{0}^{t} e^{(t-s) \Delta} f(u(s)) d s \leq \int_{0}^{t} e^{(t-s) \Delta} f(\bar{u}(s)) d s \lesssim \int_{0}^{t} e^{(t-s) \Delta} e^{w} d s .
$$

Then (3.21) shows us the desired convergence. Thus we complete the proof of Theorem 1.1 for the case $A=1$.

Remark 3.1. We explain the structure of the transformations (3.10) and (3.22) used in the proof of Theorem 1.1] for the cases $A>1$ and $A=1$, respectively. Let $f$ and $g$ satisfy (1.2) and (1.8), and define $G$ by

$$
G(v):=\int_{v}^{\infty} \frac{d s}{g(s)}
$$

Assume that $v$ satisfies $\partial_{t} v-\Delta v=g(v)$, and consider the following general transformation

$$
\tilde{u}(x, t):=F^{-1}(G(v(x, t))) .
$$

By a simple calculation we see that $\tilde{u}$ satisfies

$$
\partial_{t} \tilde{u}-\Delta \tilde{u}-f(\tilde{u})=\frac{f(\tilde{u})|\nabla v|^{2}}{g(v)^{2} F(\tilde{u})}\left(g^{\prime}(v) G(v)-f^{\prime}(\tilde{u}) F(\tilde{u})\right) .
$$


Then one can easily check that

$$
g(s)= \begin{cases}s^{\frac{A}{A-1}} & \text { if } \quad A>1, \\ e^{s} & \text { if } \quad A=1,\end{cases}
$$

is a solution of the equation $g^{\prime}(s) G(s)=A$, which implies that $G(s)=(A-1) s^{-\frac{1}{A-1}}$ for the case $A>1$ and $G(s)=e^{-s}$ for the case $A=1$. Then (3.25) corresponds to (3.10) for the case $A>1$ and (3.22) for the case $A=1$, respectively.

\section{Nonexistence of solutions for problem (1.1)}

In this section we discuss the nonexistence results of local in time nonnegative classical solutions of (1.1), and prove Theorems 1.2 and 1.3. Recall

$$
A=\lim _{s \rightarrow \infty} f^{\prime}(s) F(s) .
$$

Proof of Theorem 1.2. We first consider the case $A>1$. Let $r \in[A-1, N / 2)$. By [26, Corollary 5.1] we can take a nonnegative function $v_{0} \in L^{r /(A-1)}\left(\mathbb{R}^{N}\right) \subset L_{u l, \rho}^{r /(A-1)}\left(\mathbb{R}^{N}\right)$ such that there can not exists a local in time solution for the integral equation

$$
v(t)=e^{t \Delta} v_{0}+(A-1) \int_{0}^{t} e^{(t-s) \Delta} v(s)^{\frac{A}{A-1}} d s .
$$

Remark that, under the assumption of Theorem 1.2 for the case $A>1$, we have

$$
1 \leq \frac{r}{A-1}<\frac{N}{2}\left[\frac{A}{A-1}-1\right] .
$$

Let $s_{2}>0$ be the constant satisfying (1.18) for all $s \geq s_{2}$. Define

$$
u_{0}(x):=\max \left\{F^{-1}\left(v_{0}^{-\frac{1}{A-1}}\right), s_{2}\right\} .
$$

The proof is by contradiction. Suppose that there exist an existence time $T>0$ and a local in time nonnegative classical solution $\bar{u} \in C^{2,1}\left(\mathbb{R}^{N} \times(0, T)\right)$ for the problem (1.1) satisfying

$$
\lim _{t \rightarrow 0}\left\|u(t)-e^{t \Delta} u_{0}\right\|_{L_{u l, \rho}^{\frac{r}{A-1}}\left(\mathbb{R}^{N}\right)}=0 .
$$

Note that $F\left(u_{0}\right)^{-r} \in L_{u l, \rho}^{1}\left(\mathbb{R}^{N}\right)$. Since $\bar{u}$ is a classical solution of (1.1) satisfying (4.3), as in the proof of Proposition 2.5, we can rewrite (1.1) by the integral form

$$
\bar{u}(x, t)=e^{t \Delta} u_{0}+\int_{0}^{t} e^{(t-s) \Delta} f(\bar{u}(s)) d s .
$$

This implies $\bar{u}(x, t) \geq e^{t \Delta} u_{0} \geq s_{2}$ and

$$
f^{\prime}(\bar{u}(x, t)) F(\bar{u}(x, t)) \geq A
$$

in $\mathbb{R}^{N} \times(0, T)$. Define

$$
\bar{v}(x, t):=F(\bar{u}(x, t))^{-(A-1)} \quad \text { in } \mathbb{R}^{N} \times(0, T) .
$$

Then, by (4.5) we see that $\bar{v}$ satisfies

$$
\partial_{t} \bar{v}-\Delta \bar{v}-(A-1) \bar{v}^{\frac{A}{A-1}}=(A-1) F(\bar{u})^{-A-1} f(\bar{u})^{-2}|\nabla \bar{u}|^{2}\left[f^{\prime}(\bar{u}) F(\bar{u})-A\right] \geq 0
$$


in $\mathbb{R}^{N} \times(0, T)$. By Lemma 3.2 (i) together with (1.18) we can apply Lemma 2.4, and by (4.2) and (4.4) we have

$$
\bar{v}(x, t) \geq F\left(e^{t \Delta} u_{0}\right)^{-(A-1)} \geq e^{t \Delta}\left[F\left(u_{0}\right)^{-(A-1)}\right] \geq e^{t \Delta} v_{0} .
$$

Furthermore, for any $\tau \in(0, T)$, by (4.6) we have

$$
\bar{v}(x, t) \geq\left(e^{(t-\tau) \Delta} \bar{v}(\tau)\right)(x)+(A-1) \int_{\tau}^{t}\left[e^{(t-s) \Delta} \bar{v}(s)^{\frac{A}{A-1}}\right](x) d s
$$

in $\mathbb{R}^{N} \times(\tau, T)$. Then, since

$$
(A-1) \int_{\tau}^{t}\left[e^{(t-s) \Delta} \bar{v}(s)^{\frac{A}{A-1}}\right](x) d s \leq \bar{v}(x, t)
$$

for all $\tau \in(0, t)$ and $\bar{v}$ is nonnegative, we see that

$$
\lim _{\tau \rightarrow 0} \int_{\tau}^{t}\left[e^{(t-s) \Delta} \bar{v}(s)^{\frac{A}{A-1}}\right](x) d s=\int_{0}^{t}\left[e^{(t-s) \Delta} \bar{v}(s)^{\frac{A}{A-1}}\right](x) d s
$$

exists for all $(x, t) \in \mathbb{R}^{N} \times(0, T)$. Taking the limit $\tau \rightarrow 0$, by (4.7) and (4.8) we obtain

$$
\bar{v}(x, t) \geq e^{t \Delta} v_{0}(x)+(A-1) \int_{0}^{t}\left[e^{(t-s) \Delta} \bar{v}(s)^{\frac{A}{A-1}}\right](x) d s,
$$

that is, $\bar{v}$ is a supersolution of (4.1). Then we can construct a local in time solution $v \in$ $C^{2,1}\left(\mathbb{R}^{N} \times(0, T)\right)$ of the integral equation (4.1) with the aid of Proposition 2.1. This yields a contradiction.

Next we consider the case $A=1$. The proof is similar to the above argument, thus we only show a brief sketch of the proof. Let $r \in(0, N / 2)$. By Proposition 2.5 we can take a nonnegative function $w_{0}$ such that $e^{r w_{0}} \in L_{u l, \rho}^{1}\left(\mathbb{R}^{N}\right)$ and there can not exists a local in time solution for the integral equation

$$
w(t, x)=e^{t \Delta} w_{0}+\int_{0}^{t} e^{(t-s) \Delta} e^{w(s)} d s .
$$

Define $u_{0}(x):=\max \left\{F^{-1}\left(e^{-w_{0}(x)}\right), s_{2}\right\}$. Suppose that there exists a local in time classical solution $\bar{u} \in C^{2,1}\left(\mathbb{R}^{N} \times(0, T)\right)$ for problem (1.1) with $\left\|\bar{u}(t)-e^{t \Delta} u_{0}\right\|_{L^{\infty}} \rightarrow 0$ as $t \rightarrow 0$. As in the proof for the case $A>1, \bar{u}$ also satisfies the integral equation (4.4). Define

$$
\bar{w}(x, t):=\log F(\bar{u}(x, t))^{-1} \quad \text { in } \mathbb{R}^{N} \times(0, T) .
$$

Similarly to the case $A>1$, we have $f^{\prime}(\bar{u}) F(\bar{u}) \geq 1$ in $\mathbb{R}^{N} \times(0, T)$, and obtain

$$
\partial_{t} \bar{w}-\Delta \bar{w}-e^{\bar{w}}=\frac{\left[f^{\prime}(\bar{u}) F(\bar{u})-1\right]|\nabla \bar{u}|^{2}}{f(\bar{u})^{2} F(\bar{u})^{2}} \geq 0 .
$$

Thus, as in the above argument, by the concavity of $\log F(s)^{-1}$ in $\left(s_{2}, \infty\right)$ we see that $\bar{w}$ satisfies

$$
\bar{w}(x, t) \geq\left(e^{t \Delta} w_{0}\right)(x)+\int_{0}^{t}\left[e^{(t-s) \Delta} e^{\bar{w}(s)}\right](x) d s,
$$

and we can construct a solution of (4.9) with the help of Proposition 2.1, which yields a contradiction. Thus we complete the proof of Theorem 1.2 . 
Proof of Theorem 1.3. Let $r \in(0, N / 2)$. Put $h(s):=1-f^{\prime}(s) F(s) \geq 0$. Then we have $h(s) \rightarrow 0$ as $s \rightarrow \infty$ and

$$
(f(s) F(s))^{\prime}=f^{\prime}(s) F(s)-1=-h(s) \leq 0,
$$

hence $l:=\lim _{s \rightarrow \infty} f(s) F(s) \geq 0$ exists. Since $-h(s)$ is the derivative of $f(s) F(s)$ and $l$ exists, $h$ is integrable on $\left[s_{3}, \infty\right)$, where $s_{3}$ is the constant appearing in the assumption of Theorem 1.3 , Let $\epsilon>0$ be a sufficiently small constant. For the case $l>0$, since

$$
(\log f(s))^{\prime}=\frac{f^{\prime}(s) F(s)}{f(s) F(s)} \geq \frac{1}{l}-\epsilon
$$

for all sufficiently large $s>0$, by a simple calculation we get $f(s) \gtrsim e^{(1 / l-\epsilon) s}$ for all sufficiently large $s>0$. On the other hand, for the case $l=0$, since

$$
f(s) F(s)=\int_{s}^{\infty} h(u) d u=: \frac{1}{H(s)}, \quad(\log f(s))^{\prime}=\frac{f^{\prime}(s) F(s)}{f(s) F(s)} \geq(1-\epsilon) H(s),
$$

as in the above calculation, we obtain

$$
f(s) \gtrsim e^{(1-\epsilon) g(s)} \quad \text { with } g(s)=\int_{s_{3}}^{s} H(u) d u
$$

for all $s \geq s_{3}$. Both cases $l=0$ and $l>0$ are treated in the same manner, so we give the proof only for the case $l=0$.

Assume that, for any nonnegative initial function $u_{0}$ satisfying $F\left(u_{0}\right)^{-r} \in L_{u l, \rho}^{1}\left(\mathbb{R}^{N}\right)$, there exists a classical solution $u$ of

$$
u(t)=e^{t \Delta} u_{0}+\int_{0}^{t} e^{(t-s) \Delta} f(u(s)) d s .
$$

Then, by (4.11) we can take a constant $C>0$ such that

$$
u(t) \geq e^{t \Delta} u_{0}+\int_{0}^{t} e^{(t-s) \Delta} e^{(1-\epsilon) g(u(s))-C} d s .
$$

Remark that we can assume that $u_{0} \geq s_{3}$ without loss of generality by considering $\max \left\{u_{0}, s_{3}\right\}$ instead of $u_{0}$, and so $u \geq s_{3}$. Since

$$
g^{\prime}(s)=H(s)>0, \quad \lim _{s \rightarrow \infty} \frac{g^{\prime \prime}(s)}{\left(g^{\prime}(s)\right)^{2}}=\lim _{s \rightarrow \infty} h(s)=0,
$$

we can apply the similar calculation as in the proof of Proposition 2.5 for

$$
G(s):=\int_{s}^{\infty} \frac{d u}{e^{(1-\epsilon) g(u)-C}} .
$$

Then we obtain

$$
G(s) \gtrsim \frac{1}{g^{\prime}(s)} e^{-(1-\epsilon) g(s)} \Longleftrightarrow G(s)^{-r} \lesssim\left(g^{\prime}(s)\right)^{r} e^{(1-\epsilon) r g(s)}
$$

for all sufficiently large $s>0$. On the other hand, by (4.10) and (4.11) we have

$$
F(s)^{-r} \gtrsim\left(g^{\prime}(s)\right)^{r} e^{(1-\epsilon) r g(s)}
$$

for all sufficiently large $s>0$. These imply that $G(s)^{-r} \lesssim F(s)^{-r}$ for all sufficiently large $s>0$. In particular, we have $G\left(u_{0}\right)^{-r} \in L_{u l, \rho}^{1}\left(\mathbb{R}^{N}\right)$. Then we see that (4.12) yields a contradiction. In fact, if there exists a solution $u$ satisfying (4.12), then, in view of Proposition 2.1, we can construct a solution of

$$
u(t)=e^{t \Delta} u_{0}+\int_{0}^{t} e^{(t-s) \Delta} e^{(1-\epsilon) g(u(s))-C} d s .
$$

This contradicts Proposition 2.5 since $G\left(u_{0}\right)^{-r} \in L_{u l, \rho}^{1}\left(\mathbb{R}^{N}\right)$, and we complete the proof of Theorem 1.3 . 


\section{Applications}

In this section we apply Theorems 1.11 .3 to some examples of nonlinear heat equations. In particular, we consider the following cases: $f(u)=u^{p}+u^{q}(p>q>1)$ and $f(u)=e^{u^{2}}$.

5.1 Case $f(u)=u^{p}+u^{q}$ with $p>q>1$

Consider the case $f(u)=u^{p}+u^{q}$ with $p>q>1$, that is,

$$
\begin{cases}\partial_{t} u=\Delta u+u^{p}+u^{q}, & x \in \mathbb{R}^{N}, t>0, \\ u(x, 0)=u_{0}(x) \geq 0, & x \in \mathbb{R}^{N} .\end{cases}
$$

Before stating the existence and nonexistence results for $f(u)=u^{p}+u^{q}$, we prepare the following lemma. Recall that

$$
F(s)=\int_{s}^{\infty} \frac{d u}{u^{p}+u^{q}}
$$

Lemma 5.1. Let $p>q>1$ and $f(s)=s^{p}+s^{q}$. Then there hold the following properties.

(i) For all sufficiently large $s>0$, it holds

$$
f^{\prime}(s) F(s) \leq \lim _{s \rightarrow \infty} f^{\prime}(s) F(s)=\frac{p}{p-1} .
$$

(ii) Let $r \geq 1$. Then

$$
F(s)^{-r} \lesssim s^{r(p-1)}+s^{r(q-1)}
$$

for all $s>0$.

Theorem $5.1\left(f(u)=u^{p}+u^{q}\right)$. Let $N \geq 1$ and $p>q>1$.

(i) (Subcritical case) Let $r>0$ satisfy $r \geq 1 /(p-1)$ and $r>N / 2$ and $u_{0} \in L_{u l, \rho}^{r(p-1)}\left(\mathbb{R}^{N}\right)$ be a nonnegative function. Then there exists a local in time classical solution u for problem (5.1) satisfying

$$
\lim _{t \rightarrow 0}\left\|u(t)-e^{t \Delta} u_{0}\right\|_{L_{u l, \rho}^{r(p-1)}\left(\mathbb{R}^{N}\right)}=0 .
$$

In particular, if $u_{0} \in \mathcal{L}_{u l, \rho}^{r(p-1)}\left(\mathbb{R}^{N}\right)$, then the solution converges to the initial data in $L_{u l, \rho}^{r(p-1)}\left(\mathbb{R}^{N}\right)$, that is,

$$
\lim _{t \rightarrow 0}\left\|u(t)-u_{0}\right\|_{L_{u l, \rho}^{r(p-1)}\left(\mathbb{R}^{N}\right)}=0 .
$$

Furthermore, the existence time $T$ can be estimated to satisfy

$$
T^{\frac{N}{2 p}} \rho^{-\frac{N}{p}}+\max \left\{\left\|u_{0}\right\|_{L_{u l, \rho}^{r(p-1)}\left(\mathbb{R}^{N}\right)}^{r(p-1)}, \rho^{N}\right\}\left(T^{r-\frac{N}{2} \cdot \frac{p-1}{p}} \rho^{-N \cdot \frac{1}{p}}+T^{r-\frac{N}{2}}\right) \geq \gamma
$$

for some $\gamma>0$ depending only on $N, p, q$ and $r$.

(ii) (Critical case) Assume that $p>1+2 / N$. Let $u_{0} \in \mathcal{L}_{u l, \rho}^{\frac{N}{2}(p-1)}\left(\mathbb{R}^{N}\right)$ be a nonnegative function. Then there exists a local in time classical solution u for problem (5.1) satisfying

$$
\lim _{t \rightarrow 0}\left\|u(t)-u_{0}\right\|_{L_{u l, \rho}^{\frac{N}{2}(p-1)}\left(\mathbb{R}^{N}\right)}=0 .
$$

(iii) (Nonexistence) Let $p>1+2 / N$ and $1 /(p-1) \leq r<N / 2$. Then there exists a nonnegative initial function $u_{0} \in L_{u l, \rho}^{r(p-1)}\left(\mathbb{R}^{N}\right)$ and problem (5.1) can not possess local in time nonnegative classical solutions satisfying (5.2). 
For the proof of Theorem 5.1, we check the conditions of Theorems 1.1 and 1.2, To this end, we start from the proof of Lemma 5.1 .

Proof of Lemma 5.1. We see that $f$ is a positive convex function in $(0, \infty)$ and

$$
\begin{aligned}
f^{\prime}(s) F(s) & =\left(p s^{p-1}+q s^{q-1}\right) \int_{s}^{\infty} \frac{d u}{u^{p}+u^{q}} \\
& =\left(p s^{p-1}+q s^{q-1}\right) \int_{s}^{\infty} \frac{d u}{u^{p}\left(1+u^{q-p}\right)} \\
& =\left(p s^{p-1}+q s^{q-1}\right)\left[\frac{s^{1-p}}{(p-1)\left(1+s^{q-p}\right)}+\frac{p-q}{p-1} \int_{s}^{\infty} u^{-(p-1)} \cdot \frac{u^{q-p-1}}{\left(1+u^{q-p}\right)^{2}} d u\right] \\
& \leq \frac{p+q s^{q-p}+o\left(s^{q-p}\right)}{(p-1)\left(1+s^{q-p}\right)}<\frac{p+p s^{q-p}}{(p-1)\left(1+s^{q-p}\right)}=\frac{p}{p-1}
\end{aligned}
$$

for all sufficiently large $s>0$. Here we used

$$
\int_{s}^{\infty} u^{-(p-1)} \cdot \frac{u^{q-p-1}}{\left(1+u^{q-p}\right)^{2}} d u \leq \int_{s}^{\infty} u^{q-p-1-(p-1)} d u=O\left(s^{q-p-(p-1)}\right)=o\left(s^{q-p}\right)
$$

as $s \rightarrow \infty$. Furthermore, it is easy to check that

$$
\lim _{s \rightarrow \infty} f^{\prime}(s) F(s)=\frac{p}{p-1}=1+\frac{1}{p-1}>1 .
$$

This proves (i).

It remains to prove (ii). Since

$$
\begin{aligned}
F(s) & =\int_{s}^{\infty} \frac{d u}{u^{q}\left(1+u^{p-q}\right)}=\frac{s^{1-q}}{(q-1)\left(1+s^{p-q}\right)}-\frac{p-q}{q-1} \int_{s}^{\infty} u^{1-q} \cdot \frac{u^{p-q-1}}{\left(1+u^{p-q}\right)^{2}} d u \\
& =\frac{s^{1-q}}{q-1} \cdot \frac{1}{1+s^{p-q}}-\frac{p-q}{q-1} \int_{s}^{\infty} \frac{1}{u^{p}+u^{q}} \cdot \frac{u^{p-q}}{1+u^{p-q}} d u,
\end{aligned}
$$

we have

$$
F(s) \geq \frac{s^{1-q}}{2(q-1)}-\frac{p-q}{q-1} F(s) \Longleftrightarrow F(s) \geq \frac{1}{2(p-1)} s^{1-q}
$$

for all $s \in(0,1)$. On the other hand, since $f(s)=s^{p}+s^{q} \leq 2 s^{p}$ for $s \geq 1$, we have

$$
F(s) \geq \frac{1}{2} \int_{s}^{\infty} \frac{d u}{u^{p}}=\frac{s^{-(p-1)}}{2(p-1)}
$$

for all $s \geq 1$. Combining (5.5) and (5.6), we obtain $F(s)^{-r} \lesssim s^{r(p-1)}+s^{r(q-1)}$ for all $s>0$. This yields the assertion (ii). Thus we complete the proof of Lemma 5.1 .

Proof of Theorem 5.1. Applying Lemma 5.1, we check the conditions of Theorems 1.11.2. We first prove assertion (i). By Lemma 5.1 (ii) we have

$$
\begin{aligned}
\int_{B_{\rho}(y)} F\left(u_{0}(x)\right)^{-r} d x & =\left(\int_{B_{\rho}(y) \cap\left\{u_{0}(x)<1\right\}}+\int_{B_{\rho}(y) \cap\left\{u_{0}(x) \geq 1\right\}}\right) F\left(u_{0}(x)\right)^{-r} d x \\
& \leq F(1)^{-r}\left|B_{\rho}(0)\right|+(2(p-1))^{r} \int_{B_{\rho}(y)}\left|u_{0}(x)\right|^{r(p-1)} d x
\end{aligned}
$$

for all $y \in \mathbb{R}^{N}$. Then, since $u_{0} \in L_{u l, \rho}^{r(p-1)}\left(\mathbb{R}^{N}\right)$, we have $F\left(u_{0}\right)^{-r} \in L_{u l, \rho}^{1}\left(\mathbb{R}^{N}\right)$. By Lemma 5.1 (i) we have

$$
r \geq \frac{1}{p-1}=\lim _{s \rightarrow \infty} f^{\prime}(s) F(s)-1,
$$


and we see from Theorem 1.1 (i) that problem (5.1) has a local in time solution $u$ satisfying (5.2). Furthermore, by (1.13) and (5.7) we obtain the estimate on the existence time and prove (5.3).

We next prove assertion (ii). Since $p>1+2 / N$, by Lemma 5.1 (i) we have

$$
\frac{N}{2}>\frac{1}{p-1}=\lim _{s \rightarrow \infty} f^{\prime}(s) F(s)-1 .
$$

Assuming that $u_{0} \in \mathcal{L}_{u l, \rho}^{\frac{N}{2}(p-1)}\left(\mathbb{R}^{N}\right)$, we prove that the initial data $u_{0}$ satisfies

$$
F\left(u_{0}\right)^{-\frac{N}{2}} \in \mathcal{L}_{u l, \rho}^{1}\left(\mathbb{R}^{N}\right)
$$

Since $u_{0} \in \mathcal{L}_{u l, \rho}^{\frac{N}{2}(p-1)}\left(\mathbb{R}^{N}\right)$, there exists a sequence $\left\{u_{n}\right\} \subset B U C\left(\mathbb{R}^{N}\right)$ such that $u_{n} \rightarrow u_{0}$ in $L_{u l, \rho}^{\frac{N}{2}(p-1)}\left(\mathbb{R}^{N}\right)$ as $n \rightarrow \infty$. Then, by the Hölder inequality we have $u_{n} \rightarrow u_{0}$ in $L_{u l, \rho}^{\frac{N}{2}(q-1)}\left(\mathbb{R}^{N}\right)$. Remark that $F\left(u_{n}\right)^{-\frac{N}{2}} \in B U C\left(\mathbb{R}^{N}\right)$. Applying the mean value theorem, we have

$$
\left|F\left(u_{0}\right)^{-\frac{N}{2}}-F\left(u_{n}\right)^{-\frac{N}{2}}\right| \leq \frac{N}{2} \frac{F\left(u_{0}+\theta\left(u_{n}-u_{0}\right)\right)^{-\frac{N}{2}-1}}{f\left(u_{0}+\theta\left(u_{n}-u_{0}\right)\right)}\left|u_{0}-u_{n}\right|,
$$

where $0<\theta<1$. Let $v:=u_{0}+\theta\left(u_{n}-u_{0}\right)$. Since $f(s) \geq s^{p}$ and $f(s) \geq s^{q}$, by Lemma 5.1 (ii) we have

$$
\left|F\left(u_{0}\right)^{-\frac{N}{2}}-F\left(u_{n}\right)^{-\frac{N}{2}}\right| \lesssim\left(v^{\frac{N}{2}(p-1)-1}+v^{\frac{N}{2}(q-1)-1}\right)\left|u_{0}-u_{n}\right|
$$

Therefore it follows from the Hölder inequality that

$$
\begin{aligned}
\int_{B_{\rho}(y)}\left|F\left(u_{0}\right)^{-\frac{N}{2}}-F\left(u_{n}\right)^{-\frac{N}{2}}\right| d x & \lesssim \int_{B_{\rho}(y)}\left(v^{\frac{N}{2}(p-1)-1}+v^{\frac{N}{2}(q-1)-1}\right)\left|u_{0}-u_{n}\right| d x \\
& \lesssim\left(\int_{B_{\rho}(y)} v^{\frac{N}{2}(p-1)} d x\right)^{\frac{p-1-\frac{2}{N}}{(p-1)}}\left\|u_{0}-u_{n}\right\|_{L^{\frac{N}{2}(p-1)}\left(B_{\rho}(y)\right)} \\
& +\left(\int_{B_{\rho}(y)} v^{\frac{N}{2}(q-1)} d x\right)^{\frac{q-1-\frac{2}{N}}{(q-1)}}\left\|u_{0}-u_{n}\right\|_{L^{\frac{N}{2}(q-1)}\left(B_{\rho}(y)\right)}
\end{aligned}
$$

Then $F\left(u_{n}\right)^{-\frac{N}{2}}$ converges to $F\left(u_{0}\right)^{-\frac{N}{2}}$ as $n \rightarrow \infty$ in $L_{u l, \rho}^{1}\left(\mathbb{R}^{N}\right)$ since

$$
u_{0}, u_{n} \in L_{u l, \rho}^{\frac{N}{2}(p-1)}\left(\mathbb{R}^{N}\right) \subset L_{u l, \rho}^{\frac{N}{2}(q-1)}\left(\mathbb{R}^{N}\right) \text { and } u_{n} \rightarrow u_{0} \text { in } L_{u l, \rho}^{\frac{N}{2}(p-1)}\left(\mathbb{R}^{N}\right),
$$

and so we obtain (5.8). Therefore, by Theorem 1.1 (ii) we get a local in time solution for problem (5.1) satisfying (5.2) with $r=N / 2$. Since $u_{0} \in \mathcal{L}_{u l, \rho}^{\frac{N}{2}(p-1)}\left(\mathbb{R}^{N}\right)$, by Lemma 2.2 we see that $e^{t \Delta} u_{0}$ converges to $u_{0}$ in $L_{u l, \rho}^{\frac{N}{2}(p-1)}\left(\mathbb{R}^{N}\right)$ as $t \rightarrow 0$. Thus (5.4) holds.

We finally prove assertion (iii). For the proof of assertion (iii), we apply Theorem 1.2. Let $p>1+2 / N$. Consider

$$
\begin{cases}\partial_{t} u=\Delta u+u^{p}, & x \in \mathbb{R}^{N}, t>0 \\ u(x, 0)=u_{0}(x) \geq 0, & x \in \mathbb{R}^{N}\end{cases}
$$

Put $f_{0}(s)=s^{p}$. Then, since

$$
f_{0}^{\prime}(s) \int_{s}^{\infty} \frac{d u}{f_{0}(u)}=\frac{p}{p-1}, \quad \frac{p}{p-1}-1=\frac{1}{p-1}<\frac{N}{2},
$$


for $r \in[1 /(p-1), N / 2)$, by Theorem 1.2 we find an initial function $u_{0} \in L_{u l, \rho}^{r(p-1)}\left(\mathbb{R}^{N}\right)$ such that there can not exist nonnegative classical solutions of (5.9). See also [26, Corollary 5.1]. Suppose that there exists a classical solution $u$ of (5.1) with this initial data $u_{0}$. Then we have

$$
\partial_{t} u \geq \Delta u+u^{p}, \quad x \in \mathbb{R}^{N}, t>0, \quad u(x, 0)=u_{0}(x), \quad x \in \mathbb{R}^{N},
$$

and so $u$ is a supersolution of (5.9). Thus, as in the proof of Theorem 1.2, by Proposition 2.1 we can construct a solution of (5.9), which is a contradiction. Therefore we prove assertion (iii), and the proof of Theorem 5.1 is complete.

\subsection{Case $f(u)=e^{u^{2}}$}

Consider the case $f(u)=e^{u^{2}}$, that is,

$$
\begin{cases}\partial_{t} u=\Delta u+e^{u^{2}}, & x \in \mathbb{R}^{N}, t>0 \\ u(x, 0)=u_{0}(x) \geq 0, & x \in \mathbb{R}^{N}\end{cases}
$$

Recall that

$$
F(s)=\int_{s}^{\infty} \frac{d u}{e^{u^{2}}}
$$

We first prepare several lemmas.

Lemma 5.2. Let $f(s)=e^{s^{2}}$ and $F$ be the function defined by (5.11).

(i) It holds $F(s)^{-1} \lesssim(1+s) e^{s^{2}}$ for all $s>0$.

(ii) Let $0<\sigma \leq 1$. Define $h_{\sigma}(t):=t^{\sigma} e^{\sigma t^{2}}$ and $g_{\sigma}(s):=F\left(h_{\sigma}^{-1}(s)\right)^{-\sigma}$, where $h_{\sigma}^{-1}$ denotes the inverse function of $h_{\sigma}$. Then there holds that $\left|g_{\sigma}^{\prime}(s)\right| \lesssim 1$ for all $s>0$.

Proof. By integration by parts we have

$$
F(s)=\frac{1}{2} \int_{s}^{\infty} u^{-1}\left(-e^{-u^{2}}\right)^{\prime} d u=\frac{1}{2} s^{-1} e^{-s^{2}}-\frac{1}{2} \int_{s}^{\infty} u^{-2} e^{-u^{2}} d u
$$

for all $s>0$. Since

$$
\int_{s}^{\infty} u^{-2} e^{-u^{2}} d u=\int_{s}^{\infty} u^{-3} \cdot u e^{-u^{2}} d u \leq s^{-3} \int_{s}^{\infty} u e^{-u^{2}} d u=\frac{1}{2} s^{-3} e^{-s^{2}} \leq \frac{1}{2} s^{-1} e^{-s^{2}}
$$

for all $s \geq 1$, we obtain

$$
F(s) \geq \frac{1}{4} s^{-1} e^{-s^{2}}
$$

for all $s \geq 1$. In particular, since $F(0)>0$, we have $F(0)^{-1}<\infty$, and by (5.12) we obtain assertion (i).

We next prove assertion (ii). Put $t(s):=h_{\sigma}^{-1}(s)$. By the definition of $h_{\sigma}$, we have $s=$ $t(s)^{\sigma} e^{\sigma t(s)^{2}}$. Thus we obtain

$$
1=\left(\sigma+2 \sigma t(s)^{2}\right) t(s)^{\sigma-1} e^{\sigma t(s)^{2}} t^{\prime}(s) .
$$

This together with assertion (i) and the assumption $0<\sigma \leq 1$ implies that

$$
\left|g_{\sigma}^{\prime}(s)\right|=\sigma F(t)^{-\sigma-1} f(t)^{-1} t^{\prime}(s) \lesssim(1+t(s))^{\sigma+1} e^{\sigma t(s)^{2}} \cdot \frac{t(s)^{1-\sigma} e^{-\sigma t(s)^{2}}}{\sigma+2 \sigma t(s)^{2}} \lesssim 1
$$

for all $s>0$. This proves assertion (ii). Thus we complete the proof of Lemma 5.2 ,

With the help of Lemma 5.2, we can prove the following assertion. 
Lemma 5.3. Let $r>0$ and $h_{r}\left(u_{0}\right)=\left|u_{0}\right|^{r} e^{r\left|u_{0}(x)\right|^{2}} \in \mathcal{L}_{u l, \rho}^{1}\left(\mathbb{R}^{N}\right)$. Then $F\left(u_{0}\right)^{-r} \in \mathcal{L}_{u l, \rho}^{1}\left(\mathbb{R}^{N}\right)$.

Proof. Let $r>0$ and assume $h_{r}\left(u_{0}\right)=\left|u_{0}\right|^{r} e^{r\left|u_{0}(x)\right|^{2}} \in \mathcal{L}_{u l, \rho}^{1}\left(\mathbb{R}^{N}\right)$. Put $R:=\max \{r, 1\}$ and

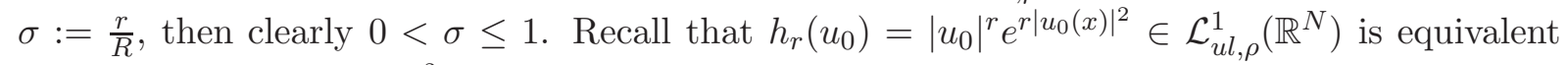
to $h_{\sigma}\left(u_{0}\right)=\left|u_{0}\right|^{\sigma} e^{\sigma\left|u_{0}(x)\right|^{2}} \in \mathcal{L}_{u l, \rho}^{R}\left(\mathbb{R}^{N}\right)$. Now define $v_{0}(x):=h_{\sigma}\left(u_{0}\right)$, then $v_{0} \in \mathcal{L}_{u l, \rho}^{R}\left(\mathbb{R}^{N}\right)$ and $g_{\sigma}\left(v_{0}(x)\right)=F\left(u_{0}(x)\right)^{-\sigma}$, where $g_{\sigma}$ is the function defined in Lemma 5.2. Since $v_{0} \in \mathcal{L}_{u l, \rho}^{R}\left(\mathbb{R}^{N}\right)$, one can take a sequence $\left\{v_{n}\right\}_{n \in \mathbb{N}} \subset B U C\left(\mathbb{R}^{N}\right)$ such that $v_{n} \rightarrow v_{0}$ in $L_{u l, \rho}^{R}\left(\mathbb{R}^{N}\right)$ as $n \rightarrow \infty$. Define $u_{n}:=g_{\sigma}\left(v_{n}\right)$, then $u_{n}$ also belongs to $B U C\left(\mathbb{R}^{N}\right)$. This together with the mean value theorem and Lemma 5.2 (ii) implies that

$$
\begin{aligned}
\sup _{y \in \mathbb{R}^{N}} \int_{B_{\rho}(y)}\left|u_{n}(x)-F\left(u_{0}(x)\right)^{-\sigma}\right|^{R} d x & =\sup _{y \in \mathbb{R}^{N}} \int_{B_{\rho}(y)}\left|g_{\sigma}\left(v_{n}(x)\right)-g_{\sigma}\left(v_{0}(x)\right)\right|^{R} d x \\
& \lesssim \sup _{y \in \mathbb{R}^{N}} \int_{B_{\rho}(y)}\left|v_{n}(x)-v_{0}(x)\right|^{R} d x \rightarrow 0
\end{aligned}
$$

as $n \rightarrow \infty$. Thus we see that $F\left(u_{0}\right)^{-\sigma} \in \mathcal{L}_{u l, \rho}^{R}\left(\mathbb{R}^{N}\right)$, which implies $F\left(u_{0}\right)^{-r} \in \mathcal{L}_{u l, \rho}^{1}\left(\mathbb{R}^{N}\right)$. Therefore we complete the proof of Lemma 5.3.

Furthermore, we introduce one lemma on the convergence of $e^{t \Delta} u_{0}$ to $u_{0}$ as $t \rightarrow 0$.

Lemma 5.4. Let $r>0$ and $h_{r}\left(u_{0}\right)=\left|u_{0}\right|^{r} e^{r\left|u_{0}\right|^{2}} \in \mathcal{L}_{u l, \rho}^{1}\left(\mathbb{R}^{N}\right)$, then

$$
\lim _{t \rightarrow 0}\left\|h_{r}\left(\left|e^{t \Delta} u_{0}-u_{0}\right|\right)\right\|_{L_{u l, \rho}^{1}\left(\mathbb{R}^{N}\right)}=0 .
$$

Proof. By the assumption we have $h_{r}\left(u_{0}\right) \in \mathcal{L}_{u l, \rho}^{1}\left(\mathbb{R}^{N}\right)$. Note that $u_{0}$ is a nonnegative function. By Lemma 2.2 we have

$$
\sup _{y \in \mathbb{R}^{N}} \int_{B_{\rho}(y)}\left|h_{r}\left(u_{0}(x+z)\right)-h_{r}\left(u_{0}(x)\right)\right| d x \rightarrow 0
$$

as $z \rightarrow 0$. Then we prove that

$$
\sup _{y \in \mathbb{R}^{N}} \int_{B_{\rho}(y)} h_{r}\left(\left|u_{0}(x+z)-u_{0}(x)\right|\right) d x \rightarrow 0
$$

as $z \rightarrow 0$. For the proof of (5.14), we first assume that $r \geq 1$. Let $s \geq t \geq 0$. Then, by an elementary inequality

$$
|s-t|^{p} \leq\left|s^{p}-t^{p}\right| \text { for } s, t \geq 0, \quad p \geq 1
$$

we have

$$
h_{r}(|s-t|)=\sum_{n=0}^{\infty} \frac{r^{n}}{n !}(s-t)^{2 n+r} \leq \sum_{n=0}^{\infty} \frac{r^{n}}{n !}\left(s^{2 n+r}-t^{2 n+r}\right)=h_{r}(s)-h_{r}(t) .
$$

Similarly, we obtain $h_{r}(|s-t|) \leq h_{r}(t)-h_{r}(s)$ for $t \geq s \geq 0$. Thus we have $h_{r}(|s-t|) \leq$ $\left|h_{r}(s)-h_{r}(t)\right|$ for all $s, t \geq 0$. This together with (5.13) gives (5.14). Next we consider the case $0<r<1$. Put $\tilde{h}(s):=h_{r}(s)-s^{r}=s^{r}\left(e^{r s^{2}}-1\right)$ and $\hat{h}(s):=h_{r}\left(s^{1 / r}\right)=s e^{r s^{2 / r}}$. Then we have $\hat{h}^{\prime}(s)=e^{r s^{2 / r}}+2 s^{2 / r} e^{r s^{2 / r}} \geq 1$, and by the mean value theorem we obtain $|\hat{h}(s)-\hat{h}(t)|=\left|\hat{h}^{\prime}(\theta)\right||s-t| \geq|s-t|$ for all $s, t \geq 0$, where $\theta \in(0,1)$. This is equivalent to $\left|s^{r}-t^{r}\right| \leq\left|h_{r}(s)-h_{r}(t)\right|$. Then we have

$$
\int_{B_{\rho}(y)}\left|u_{0}(x+z)^{r}-u_{0}(x)^{r}\right| d x \leq \int_{B_{\rho}(y)}\left|h_{r}\left(u_{0}(x+z)\right)-h_{r}\left(u_{0}(x)\right)\right| d x
$$


for all $y, z \in \mathbb{R}^{N}$. On the other hand, similarly to the calculation for $r \geq 1$, we see that $\tilde{h}(|s-t|) \leq|\tilde{h}(s)-\tilde{h}(t)|$ for all $s, t \geq 0$. Therefore, since $\tilde{h}(s)=h_{r}(s)-s^{r}$, by (5.15) we obtain

$$
\begin{aligned}
\int_{B_{\rho}(y)} & \tilde{h}\left(\left|u_{0}(x+z)-u_{0}(x)\right|\right) d x \leq \int_{B_{\rho}(y)}\left|\tilde{h}\left(u_{0}(x+z)\right)-\tilde{h}\left(u_{0}(x)\right)\right| d x \\
\leq & \int_{B_{\rho}(y)}\left|h_{r}\left(u_{0}(x+z)\right)-h_{r}\left(u_{0}(x)\right)\right| d x+\int_{B_{\rho}(y)}\left|u_{0}(x+z)^{r}-u_{0}(x)^{r}\right| d x \\
\leq & 2 \int_{B_{\rho}(y)}\left|h_{r}\left(u_{0}(x+z)\right)-h_{r}\left(u_{0}(x)\right)\right| d x .
\end{aligned}
$$

Furthermore, by the Hölder inequality we obtain

$$
\int_{B_{\rho}(y)}|u(x)-v(x)|^{r} d x \leq\left|B_{\rho}(0)\right|^{\frac{2}{r+2}}\left(\int_{B_{\rho}(y)}|u(x)-v(x)|^{r+2} d x\right)^{\frac{r}{r+2}}
$$

for all $y \in \mathbb{R}^{N}$ and suitable measurable functions $u, v$. This together with $\tilde{h}(s) \geq r s^{r+2}$ implies that

$$
\int_{B_{\rho}(y)}|u(x)-v(x)|^{r} d x \leq\left|B_{\rho}(0)\right|^{\frac{2}{r+2}}\left(\frac{1}{r} \int_{B_{\rho}(y)} \tilde{h}(|u(x)-v(x)|) d x\right)^{\frac{r}{r+2}} .
$$

Then, since $h_{r}(s)=\tilde{h}(s)+s^{r}$, by (5.13), (5.16) and (5.17) with $u=u_{0}(\cdot+z), v=u_{0}$ we obtain (5.14).

Once we get (5.14), we can easily prove the lemma. We give the proof only for the case $0<r<1$. Since $\tilde{h}$ is a convex function and

$$
\left(e^{t \Delta} u_{0}\right)(x)-u_{0}(x)=(4 \pi)^{-\frac{N}{2}} \int_{\mathbb{R}^{N}} e^{-\frac{|w|^{2}}{4}}\left(u_{0}(x+\sqrt{t} w)-u_{0}(x)\right) d w
$$

by the Jensen inequality and the Fubini theorem we have

$$
\int_{B_{\rho}(y)} \tilde{h}\left(\left|e^{t \Delta} u_{0}-u_{0}\right|\right) d x \leq(4 \pi)^{-\frac{N}{2}} \int_{\mathbb{R}^{N}} e^{-\frac{|w|^{2}}{4}} \int_{B_{\rho}(y)} \tilde{h}\left(\left|u_{0}(x+\sqrt{t} w)-u_{0}(x)\right|\right) d x d w
$$

for all $y \in \mathbb{R}^{N}$ and $t>0$. Furthermore, by (5.17) with $u=e^{t \Delta} u_{0}$ and $v=u_{0}$ we have

$$
\int_{B_{\rho}(y)}\left|e^{t \Delta} u_{0}-u_{0}\right|^{r} d x \leq\left|B_{\rho}(0)\right|^{\frac{2}{r+2}}\left(\frac{1}{r} \int_{B_{\rho}(y)} \tilde{h}\left(\left|e^{t \Delta} u_{0}-u_{0}\right|\right) d x\right)^{\frac{r}{r+2}} .
$$

Since $h_{r}(s)=\tilde{h}(s)+s^{r}$ and $\tilde{h}(s) \leq h_{r}(s)$, by (5.14), (5.18) and (5.19) we obtain the desired convergence and complete the proof of Lemma 5.4.

We are ready to state the results on existence and nonexistence of solutions for problem (5.10).

Theorem $5.2\left(f(u)=e^{u^{2}}\right)$. Let $N \geq 1$.

(i) (Subcritical case) For any $r>N / 2$ and nonnegative initial function $u_{0}$ satisfying

$$
\left|u_{0}\right|^{r} e^{r\left|u_{0}\right|^{2}} \in L_{u l, \rho}^{1}\left(\mathbb{R}^{N}\right),
$$

there exists a local in time classical solution u for problem (5.10) such that

$$
\lim _{t \rightarrow 0}\left\|u(t)-e^{t \Delta} u_{0}\right\|_{L^{\infty}\left(\mathbb{R}^{N}\right)}=0
$$


In addition, if $\left|u_{0}\right|^{r} e^{r\left|u_{0}\right|^{2}} \in \mathcal{L}_{u l, \rho}^{1}\left(\mathbb{R}^{N}\right)$, then

$$
\lim _{t \rightarrow 0} \sup _{y \in \mathbb{R}^{N}} \int_{B_{\rho}(y)}\left|u(x, t)-u_{0}(x)\right|^{r} e^{r\left|u(x, t)-u_{0}(x)\right|^{2}} d x=0 .
$$

Furthermore, for any sufficiently small $\epsilon>0$, the existence time $T$ can be chosen to satisfy

$$
T^{\frac{\epsilon N}{2}} \rho^{-\epsilon N}+\gamma_{\epsilon} \max \left\{\left\|u_{0}^{r} e^{r u_{0}^{2}}\right\|_{L_{u l, \rho}^{1}\left(\mathbb{R}^{N}\right)}, \rho^{N}\right\}\left(T^{r-\frac{N}{2}(1-\epsilon)} \rho^{-\epsilon N}+T^{r-\frac{N}{2}}\right) \geq \gamma
$$

where $\gamma$ depends only on $N$ and $r$, and $\gamma_{\epsilon}$ is a positive constant depending only on $N, r$ and $\epsilon$ satisfying $\gamma_{\epsilon} \rightarrow \infty$ as $\epsilon \rightarrow 0$.

(ii) (Critical case) Let $u_{0}$ be a nonnegative initial function such that $\left|u_{0}\right|^{\frac{N}{2}} e^{\frac{N}{2}\left|u_{0}\right|^{2}} \in \mathcal{L}_{u l, \rho}^{1}\left(\mathbb{R}^{N}\right)$. Then there exists a local in time classical solution for problem (5.10) satisfying (5.20) and (5.21) with $r=N / 2$.

(iii) (Nonexistence) Let $0<r<N / 2$. Then there exists a nonnegative initial function $u_{0}$ such that $\left|u_{0}\right|^{r} e^{r\left|u_{0}\right|^{2}} \in L_{u l, \rho}^{1}\left(\mathbb{R}^{N}\right)$ and problem (5.10) with (5.20) can not possess local in time nonnegative classical solutions.

Proof. We first prove assertion (i). Let $f(u)=e^{u^{2}}$. Then we have

$$
f^{\prime}(s) F(s)=2 s e^{s^{2}} \int_{s}^{\infty} \frac{d u}{e^{u^{2}}} \leq e^{s^{2}} \int_{s}^{\infty} 2 u e^{-u^{2}} d u=1
$$

for all $s>0$. Furthermore, we have $\lim _{s \rightarrow \infty} f^{\prime}(s) F(s)=1$. For $r>N / 2$, let $u_{0}$ satisfy $\left|u_{0}\right|^{r} e^{r\left|u_{0}\right|^{2}} \in$ $L_{u l, \rho}^{1}\left(\mathbb{R}^{N}\right)$. Then, by (5.12) we have

$$
\sup _{y \in \mathbb{R}^{N}} \int_{B_{\rho}(y)} F\left(u_{0}(x)\right)^{-r} d x \leq F(1)^{-r}\left|B_{\rho}(0)\right|+4^{r} \sup _{y \in \mathbb{R}^{N}} \int_{B_{\rho}(y)}\left|u_{0}(x)\right|^{r} e^{r\left|u_{0}(x)\right|^{2}} d x<\infty .
$$

Then we can apply Theorem 1.1 (i) to problem (5.10), and obtain local in time existence of a classical solution for (5.10) satisfying (5.20). The convergence (5.21) follows directly from Lemma 5.4. The estimate of the existence time (5.22) follows from (1.15).

We next prove assertion (ii). If $\left|u_{0}\right|^{\frac{N}{2}} e^{\frac{N}{2}\left|u_{0}\right|^{2}} \in \mathcal{L}_{u l, \rho}^{1}\left(\mathbb{R}^{N}\right)$, then by Lemma 5.3 we have $F\left(u_{0}\right)^{-\frac{N}{2}} \in \mathcal{L}_{u l, \rho}^{1}\left(\mathbb{R}^{N}\right)$. Thus, by Theorem 1.1 (ii) we can obtain a local in time classical solution for (5.10) satisfying (5.20). We also obtain (5.21) with $r=N / 2$ by Lemma 5.4.

We finally prove assertion (iii). Let $r \in(0, N / 2)$. By (5.23) we have $f^{\prime}(s) F(s) \leq 1$ for all $s>0$. Then, by Theorem 1.3 we can take a initial function $u_{0}$ satisfying $F\left(u_{0}\right)^{-r} \in L_{u l, \rho}^{1}\left(\mathbb{R}^{N}\right)$ such that there can not exist a solution for problem (5.10) with (5.20). On the other hand, by (5.23) we have $F(s)^{-r} \geq 2^{r} s^{r} e^{r s^{2}}$ for all $s>0$. This implies that $\left|u_{0}\right|^{r} e^{r\left|u_{0}\right|^{2}} \in L_{u l, \rho}^{1}\left(\mathbb{R}^{N}\right)$, and we complete the proof of Theorem 5.2 .

\section{A Local in time existence of solutions for a semilinear heat equa- tion in the uniformly local $L^{r}$ spaces}

Consider a semilinear heat equation

$$
\begin{cases}\partial_{t} u=\Delta u+|u|^{p-1} u, & x \in \mathbb{R}^{N}, t>0, \\ u(x, 0)=u_{0}(x), & x \in \mathbb{R}^{N},\end{cases}
$$


where $p>1$. In this section we consider the case $u_{0}$ belongs to a uniformly local $L^{r}$ space, and study existence of local in time solutions of the integral equation

$$
u(t)=e^{t \Delta} u_{0}+\int_{0}^{t} e^{(t-s) \Delta}|u(s)|^{p-1} u(s) d s .
$$

In particular, we prove Propositions 2.2 and 2.3 . These propositions can be proved by the similar argument as in [26] with the aid of Lemma 2.1. More precisely, we prove that the map $\Phi$ defined by

$$
\Phi(u):=e^{t \Delta} u_{0}+\int_{0}^{t} e^{(t-s) \Delta}|u(s)|^{p-1} u(s) d s,
$$

is a contraction map from a suitable Banach space to itself.

Proof of Proposition 2.2. Let $r \geq 1, r>\frac{N}{2}(p-1)$ and $u_{0} \in L_{u l, \rho}^{r}\left(\mathbb{R}^{N}\right)$. Define

$$
X_{M, T}:=\left\{\begin{array}{c}
u \in L^{\infty}\left(0, T ; L_{u l, \rho}^{r}\left(\mathbb{R}^{N}\right)\right) \cap L_{l o c}^{\infty}\left((0, T) ; L_{u l, \rho}^{p r}\left(\mathbb{R}^{N}\right)\right): \\
\sup _{0<t<T}\|u\|_{L_{u l, \rho}^{r}\left(\mathbb{R}^{N}\right)} \leq M, \sup _{0<t<T} t^{\alpha}\|u(t)\|_{L_{u l, \rho}^{p r}\left(\mathbb{R}^{N}\right)} \leq M
\end{array}\right\},
$$

equipped with the metric $d_{X}(u, v):=\sup \left\{t^{\alpha}\|u(t)-v(t)\|_{L_{u l, \rho}^{p r}\left(\mathbb{R}^{N}\right)}: 0<t<T\right\}$, where $\alpha:=$ $\frac{N}{2}\left(\frac{1}{r}-\frac{1}{p r}\right)$ and $M, T$ are positive constants to be chosen later. Remark that $\alpha p<1$. For $u \in X_{M, T}$, by Lemma 2.1 we have

$$
\begin{aligned}
\|\Phi(u)\|_{L_{u l, \rho}^{r}\left(\mathbb{R}^{N}\right)} & \leq\left\|u_{0}\right\|_{L_{u l, \rho}^{r}\left(\mathbb{R}^{N}\right)}+\int_{0}^{t}\|u(s)\|_{L_{u l, \rho}^{p r}\left(\mathbb{R}^{N}\right)}^{p} d s \\
& \leq\left\|u_{0}\right\|_{L_{u l, \rho}^{r}\left(\mathbb{R}^{N}\right)}+\int_{0}^{t} s^{-\alpha p} d s \cdot M^{p} \leq\left\|u_{0}\right\|_{L_{u l, \rho}^{r}\left(\mathbb{R}^{N}\right)}+\frac{T^{1-\alpha p}}{1-\alpha p} M^{p} .
\end{aligned}
$$

Again by Lemma 2.1] we obtain

$$
\begin{aligned}
& t^{\alpha}\|\Phi(u)\|_{L_{u l, \rho}^{p r}\left(\mathbb{R}^{N}\right)} \\
& \leq C\left(t^{\alpha} \rho^{-2 \alpha}+1\right)\left\|u_{0}\right\|_{L_{u l, \rho}^{r}\left(\mathbb{R}^{N}\right)}+C t^{\alpha} \int_{0}^{t}\left(\rho^{-2 \alpha}+(t-s)^{-\alpha}\right)\|u(s)\|_{L_{u l, \rho}^{p r}\left(\mathbb{R}^{N}\right)}^{p} d s \\
& \leq C\left(t^{\alpha} \rho^{-2 \alpha}+1\right)\left\|u_{0}\right\|_{L_{u l, \rho}^{r}\left(\mathbb{R}^{N}\right)}+C t^{\alpha} \int_{0}^{t}\left(\rho^{-2 \alpha}+(t-s)^{-\alpha}\right) s^{-\alpha p} d s \cdot M^{p} \\
& \leq C\left(T^{\alpha} \rho^{-2 \alpha}+1\right)\left\|u_{0}\right\|_{L_{u l, \rho}^{r}\left(\mathbb{R}^{N}\right)}+C\left(\frac{T^{1-\alpha p+\alpha}}{1-\alpha p} \rho^{-2 \alpha}+B(1-\alpha, 1-\alpha p) T^{1-\alpha p}\right) M^{p},
\end{aligned}
$$

where $B(\cdot, \cdot)$ is the beta function and $C>0$ is a constant depending only on $N, p$ and $r$. Set $M=(2 C+4)\left\|u_{0}\right\|_{L_{u l, \rho}^{r}\left(\mathbb{R}^{N}\right)}$. Let $T_{0}$ be the constant satisfying

$$
\begin{aligned}
& C T_{0}^{\alpha} \rho^{-2 \alpha}+(2 C+4)^{p}\left\|u_{0}\right\|_{L_{u l, \rho}^{r}\left(\mathbb{R}^{N}\right)}^{p-1} \cdot \frac{T_{0}^{1-\alpha p}}{1-\alpha p} \\
& \quad+p C(2 C+4)^{p}\left\|u_{0}\right\|_{L_{u l, \rho}^{r}\left(\mathbb{R}^{N}\right)}^{p-1}\left(\frac{T_{0}^{1-\alpha p+\alpha}}{1-\alpha p} \rho^{-2 \alpha}+B(1-\alpha, 1-\alpha p) T_{0}^{1-\alpha p}\right)=1 .
\end{aligned}
$$

Then $\Phi$ is a map from $X_{M, T_{0}}$ to itself. Similarly, for $u, v \in X_{M, T}$, we have

$$
\begin{aligned}
& t^{\alpha}\|\Phi(u)-\Phi(v)\|_{L_{u l, \rho}^{p r}\left(\mathbb{R}^{N}\right)} \\
& \quad \leq C p t^{\alpha} \int_{0}^{t}\left(\rho^{-2 \alpha}+(t-s)^{-\alpha}\right)\|u(s)-v(s)\|_{L_{u l, \rho}^{p r}}\left(\|u(s)\|_{L_{u l, \rho}^{p r}}^{p-1}+\|v(s)\|_{L_{u l, \rho}^{p r}}^{p-1}\right) d s
\end{aligned}
$$




$$
\leq 2 C p\left(\frac{T^{1-\alpha p+\alpha}}{1-\alpha p} \rho^{-2 \alpha}+B(1-\alpha, 1-\alpha p) T^{1-\alpha p}\right) M^{p-1} \sup _{0<t<T} t^{\alpha}\|u-v\|_{L_{u l, \rho}^{p r}\left(\mathbb{R}^{N}\right)}
$$

for the same constant $C$ as in A.2. Hence, by (A.2) wee see that $\Phi$ is a contraction map from $X_{M, T_{0}}$ to itself. Therefore, by the contraction mapping theorem we find a fixed point $u \in X_{M, T_{0}}$. Let $T$ be the maximal existence time such that the fixed point can be found in $X_{M, T}$. Then we clearly have $T \geq T_{0}$, and obtain (2.11) by (A.2).

Next we prove $u \in C\left((0, T) ; L_{u l, \rho}^{r}\left(\mathbb{R}^{N}\right)\right)$. Recall that $|u|^{p} \in L^{1}\left(0, T ; L_{u l, \rho}^{r}\left(\mathbb{R}^{N}\right)\right)$ since $u \in$ $X_{M, T}$, so $\|u\|_{L_{u l, \rho}^{p r}\left(\mathbb{R}^{N}\right)}^{p} \leq M t^{-\alpha p}$ for all $t \in(0, T)$. This together with the fact $-\alpha p>-1$ implies that

$$
u(t)-e^{t \Delta} u_{0}=\int_{0}^{t} e^{(t-s) \Delta}|u(s)|^{p-1} u(s) d s \in C\left([0, T) ; \mathcal{L}_{u l, \rho}^{r}\left(\mathbb{R}^{N}\right)\right) .
$$

On the other hand, since $e^{t \Delta} u_{0} \in \mathcal{L}_{u l, \rho}^{r}\left(\mathbb{R}^{N}\right)$, we have $e^{t \Delta} u_{0} \in C\left((0, T) ; \mathcal{L}_{u l, \rho}^{r}\left(\mathbb{R}^{N}\right)\right)$. Therefore we obtain $u \in C\left((0, T) ; \mathcal{L}_{u l, \rho}^{r}\left(\mathbb{R}^{N}\right)\right)$. We remark that, if $u_{0} \in \mathcal{L}_{u l, \rho}^{r}\left(\mathbb{R}^{N}\right)$, then $e^{t \Delta} u_{0} \in$ $C\left([0, T) ; \mathcal{L}_{u l, \rho}^{r}\left(\mathbb{R}^{N}\right)\right)$, so $u \in C\left([0, T) ; \mathcal{L}_{u l, \rho}^{r}\left(\mathbb{R}^{N}\right)\right)$.

Finally, applying the same iteration argument as in the proof of Proposition 2.3 with $q_{1}=p r$, instead of $q_{1}=q$, we obtain $L_{l o c}^{\infty}\left((0, T) ; L^{\infty}\left(\mathbb{R}^{N}\right)\right)$. This can be shown similarly, so we give its proof only for the critical case. See the argument below. Then the standard regularity argument implies that the fixed point $u$ is a classical solution of (A.11). This solution satisfies $\left\|u(t)-e^{t \Delta} u_{0}\right\|_{L_{u l, \rho}^{r}\left(\mathbb{R}^{N}\right)} \rightarrow 0$ as $t \rightarrow 0$, which follows from A.3). Thus we complete the proof of Proposition 2.2

Proof of Proposition 2.3. Assume that $r=\frac{N}{2}(p-1)>1$ and $u_{0} \in \mathcal{L}_{u l, \rho}^{r}\left(\mathbb{R}^{N}\right)$. Let $q$ satisfy $\max \{p, r\}<q<p r$ and put $\sigma:=\frac{N}{2}\left(\frac{1}{r}-\frac{1}{q}\right)$. For any $M>0$ and $T>0$, define

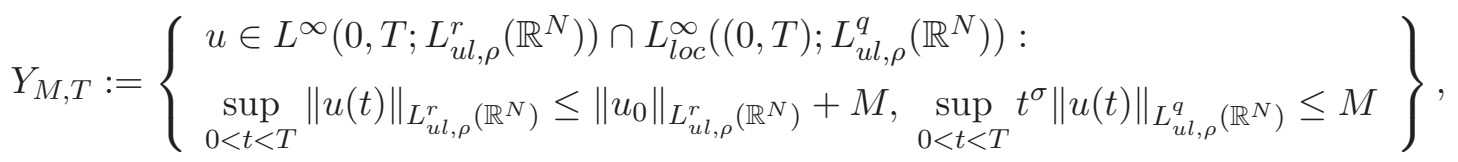

equipped with the metric $d_{Y}(u, v):=\sup \left\{t^{\sigma}\|u(t)-v(t)\|_{L_{u l, \rho}^{q}\left(\mathbb{R}^{N}\right)}: 0<t<T\right\}$. Then $\left(Y_{M, T}, d_{Y}\right)$ is a complete metric space. We show that $\Phi$ is a contraction map from $Y_{M, T}$ to itself for suitable $M>0$ and $T>0$. Let $u \in Y_{M, T}$. Lemma 2.1 shows

$$
\begin{aligned}
& \|\Phi(u)\|_{L_{u l, \rho}^{r}\left(\mathbb{R}^{N}\right)} \leq\left\|u_{0}\right\|_{L_{u l, \rho}^{r}\left(\mathbb{R}^{N}\right)}+\int_{0}^{t}\left\|e^{(t-s) \Delta}|u(s)|^{p}\right\|_{L_{u l, \rho}^{r}\left(\mathbb{R}^{N}\right)} d s \\
& \leq\left\|u_{0}\right\|_{L_{u l, \rho}^{r}\left(\mathbb{R}^{N}\right)}+C_{1} \int_{0}^{t}\left(\rho^{-N\left(\frac{1}{r}-\frac{p}{q}\right)}+(t-s)^{-\frac{N}{2}\left(\frac{1}{r}-\frac{p}{q}\right)}\right)\|u(s)\|_{L_{u l, \rho}^{q}}^{p}\left(\mathbb{R}^{N}\right) \\
& \leq\left\|u_{0}\right\|_{L_{u l, \rho}^{r}\left(\mathbb{R}^{N}\right)} d s \\
& \quad+C_{1}\left(t^{1-\sigma p} \rho^{-N\left(\frac{1}{r}-\frac{p}{q}\right)}+t^{1-\sigma p-\frac{N}{2}\left(\frac{1}{r}-\frac{p}{q}\right)} \int_{0}^{1}(1-s)^{-\frac{N}{2}\left(\frac{1}{r}-\frac{p}{q}\right)} s^{-\sigma p} d s\right) M^{p}
\end{aligned}
$$

for some constant $C_{1}>0$. Then, since $-\sigma p>-1$ and $1-\sigma p-\frac{N}{2}\left(\frac{1}{r}-\frac{p}{q}\right)=0$, we have

$$
\sup _{0<t<T}\|\Phi(u)\|_{L_{u l, \rho}^{r}\left(\mathbb{R}^{N}\right)} \leq\left\|u_{0}\right\|_{L_{u l, \rho}^{r}\left(\mathbb{R}^{N}\right)}+C_{2}\left(T^{1-\sigma p} \rho^{-N\left(\frac{1}{r}-\frac{p}{q}\right)}+1\right) M^{p}
$$


for some constant $C_{2}>0$. Similarly, we have

$$
\begin{aligned}
t^{\sigma} \| & \Phi(u)\left\|_{L_{u l, \rho}^{q}\left(\mathbb{R}^{N}\right)} \leq t^{\sigma}\right\| e^{t \Delta} u_{0}\left\|_{L_{u l, \rho}^{q}\left(\mathbb{R}^{N}\right)}+t^{\sigma} \int_{0}^{t}\right\| e^{(t-s) \Delta}|u(s)|^{p} \|_{L_{u l, \rho}^{q}\left(\mathbb{R}^{N}\right)} d s \\
\lesssim & t^{\sigma}\left\|e^{t \Delta} u_{0}\right\|_{L_{u l, \rho}^{q}\left(\mathbb{R}^{N}\right)}+t^{\sigma} \int_{0}^{t}\left(\rho^{-N\left(\frac{p}{q}-\frac{1}{q}\right)}+(t-s)^{-\frac{N}{2}\left(\frac{p}{q}-\frac{1}{q}\right)}\right)\|u(s)\|_{L_{u l, \rho}^{q}\left(\mathbb{R}^{N}\right)}^{p} d s \\
\lesssim & t^{\sigma}\left\|e^{t \Delta} u_{0}\right\|_{L_{u l, \rho}^{q}\left(\mathbb{R}^{N}\right)} \\
& +\left(t^{\sigma+1-\sigma p} \rho^{-N\left(\frac{p}{q}-\frac{1}{q}\right)}+t^{\sigma+1-\sigma p-\frac{N}{2}\left(\frac{p}{q}-\frac{1}{q}\right)} \int_{0}^{1}(1-s)^{-\frac{N}{2}\left(\frac{p}{q}-\frac{1}{q}\right)} s^{-\sigma p} d s\right) M^{p} .
\end{aligned}
$$

Then, since $-\sigma p>-1,-\frac{N}{2}\left(\frac{p}{q}-\frac{1}{q}\right)>-1$ and $\sigma+1-\sigma p-\frac{N}{2}\left(\frac{p}{q}-\frac{1}{q}\right)=0$, we obtain

$$
\sup _{0<t<T} t^{\sigma}\|\Phi(u)\|_{L_{u l, \rho}^{q}\left(\mathbb{R}^{N}\right)} \lesssim \sup _{0<t<T} t^{\sigma}\left\|e^{t \Delta} u_{0}\right\|_{L_{u l, \rho}^{q}\left(\mathbb{R}^{N}\right)}+\left(T^{1+\sigma-\sigma p} \rho^{-N\left(\frac{p}{q}-\frac{1}{q}\right)}+1\right) M^{p} .
$$

It remains to estimate the linear term $t^{\sigma}\left\|e^{t \Delta} u_{0}\right\|_{L_{u l, \rho}^{q}\left(\mathbb{R}^{N}\right)}$. Since $u_{0} \in \mathcal{L}_{u l, \rho}^{r}\left(\mathbb{R}^{N}\right)$, there exists a sequence $\left\{u_{n}\right\} \subset B U C\left(\mathbb{R}^{N}\right)$ such that $\left\|u_{0}-u_{n}\right\|_{L_{u l, \rho}^{r}\left(\mathbb{R}^{N}\right)} \rightarrow 0$ as $n \rightarrow \infty$, which yields

$$
\begin{aligned}
\sup _{0<t<T} t^{\sigma}\left\|e^{t \Delta} u_{0}\right\|_{L_{u l, \rho}^{q}\left(\mathbb{R}^{N}\right)} & \leq \sup _{0<t<T} t^{\sigma}\left\|e^{t \Delta}\left(u_{0}-u_{n}\right)\right\|_{L_{u l, \rho}^{q}\left(\mathbb{R}^{N}\right)}+\sup _{0<t<T} t^{\sigma}\left\|e^{t \Delta} u_{n}\right\|_{L_{u l, \rho}^{q}\left(\mathbb{R}^{N}\right)} \\
& \lesssim\left(T^{\sigma} \rho^{-2 \sigma}+1\right)\left\|u_{0}-u_{n}\right\|_{L_{u l, \rho}^{r}\left(\mathbb{R}^{N}\right)}+T^{\sigma}\left\|u_{n}\right\|_{L_{u l, \rho}^{q}\left(\mathbb{R}^{N}\right)}
\end{aligned}
$$

for sufficiently large $n$ and small $T>0$. Combining this inequality with (A.4) and (A.5), we obtain

$$
\sup _{0<t<T}\|\Phi(u)\|_{L_{u l, \rho}^{r}} \leq\left\|u_{0}\right\|_{L_{u l, \rho}^{r}}+M \quad \text { and } \sup _{0<t<T} t^{\sigma}\|\Phi(u)\|_{L_{u l, \rho}^{q}} \leq M
$$

for suitable $M>0$ and $T>0$. Similarly, we get

$$
\begin{aligned}
\sup _{0<t<T} t^{\sigma}\|\Phi(u)-\Phi(v)\|_{L_{u l, \rho}^{q}\left(\mathbb{R}^{N}\right)} & \\
& \lesssim\left(T^{1+\sigma-\sigma p} \rho^{-N\left(\frac{p}{q}-\frac{1}{q}\right)}+1\right) M^{p-1} \sup _{0<t<T} t^{\sigma}\|u-v\|_{L_{u l, \rho}^{q}\left(\mathbb{R}^{N}\right)}
\end{aligned}
$$

for all $u, v \in Y_{M, T}$. This proves that $\Phi$ is a contraction map from $Y_{M, T}$ to itself for sufficiently small $M>0$ and $T>0$. Thus, by the contraction mapping theorem we can find a fixed point $u \in Y_{M, T}$.

Let us prove that $u \in Y_{M, T}$ satisfies $u \in C\left((0, T) ; \mathcal{L}_{u l, \rho}^{q}\left(\mathbb{R}^{N}\right)\right)$ and $t^{\sigma}\|u\|_{L_{u l, \rho}^{q}\left(\mathbb{R}^{N}\right)} \rightarrow 0$ as $t \rightarrow 0$. Put

$$
K:=Y_{M, T} \cap\left\{u \in C\left((0, T) ; \mathcal{L}_{u l, \rho}^{q}\left(\mathbb{R}^{N}\right)\right): \lim _{t \rightarrow 0} t^{\sigma}\|u\|_{L_{u l, \rho}^{q}\left(\mathbb{R}^{N}\right)}=0\right\} .
$$

Then $K$ equipped with a metric $d_{Y}$ is a complete metric space. We now prove that $\Phi$ is a map from $K$ to $K$ (then the contraction mapping argument works in $K$, therefore the fixed point $u$ belongs to $K)$. Assume that $u_{0} \in \mathcal{L}_{u l, \rho}^{r}\left(\mathbb{R}^{N}\right)$. By the smoothing effect of the heat semigroup $e^{t \Delta}$ we have $e^{t \Delta} u_{0} \in \mathcal{L}_{u l, \rho}^{q}\left(\mathbb{R}^{N}\right) \subset \mathcal{L}_{u l, \rho}^{r}\left(\mathbb{R}^{N}\right)$ for $t>0$. This and Lemma 2.2 imply that $\left\|e^{(t+h) \Delta} u_{0}-e^{t \Delta} u_{0}\right\|_{L_{u l, \rho}^{q}\left(\mathbb{R}^{N}\right)} \rightarrow 0$ as $h \rightarrow 0$ for all $t>0$. Moreover, by the same argument as in the above argument we have $t^{\sigma}\left\|e^{t \Delta} u_{0}\right\|_{L_{u l, \rho}^{q}\left(\mathbb{R}^{N}\right)} \rightarrow 0$ as $t \rightarrow 0$. Thus we obtain $e^{t \Delta} u_{0} \in$ $K$. Since $K \cap C\left((0, T) ; B U C\left(\mathbb{R}^{N}\right)\right)$ is dense in $K$ equipped with $d_{Y}$, there exists a sequence $\left\{u_{n}\right\} \subset K \cap C\left((0, T) ; B U C\left(\mathbb{R}^{N}\right)\right)$ such that $d_{Y}\left(u, u_{n}\right) \rightarrow 0$ as $n \rightarrow \infty$. This together with the facts $u, u_{n} \in Y_{M, T}$ and (A.6) yields $d_{Y}\left(\Phi(u), \Phi\left(u_{n}\right)\right) \rightarrow 0$ as $n \rightarrow \infty$. Furthermore, we have 
$\Phi\left(u_{n}\right) \in K$, which follows from the fact $e^{t \Delta} u_{0} \in K$ and $u_{n} \in C\left((0, T) ; B U C\left(\mathbb{R}^{N}\right)\right)$. Therefore, since $K$ is a complete metric space equipped with $d_{Y}$, we obtain $\Phi(u) \in K$.

We are in position to prove $u \in C\left([0, T) ; \mathcal{L}_{u l, \rho}^{r}\left(\mathbb{R}^{N}\right)\right)$. It suffices to prove

$$
\lim _{t \rightarrow 0}\left\|u(t)-u_{0}\right\|_{L_{u l, \rho}^{r}\left(\mathbb{R}^{N}\right)}=0
$$

since $K \subset C\left((0, T) ; \mathcal{L}_{u l, \rho}^{r}\left(\mathbb{R}^{N}\right)\right)$. We clearly see that $\left\|e^{t \Delta} u_{0}-u_{0}\right\|_{L_{u l, \rho}^{r}\left(\mathbb{R}^{N}\right)} \rightarrow 0$ as $t \rightarrow 0$ by $u_{0} \in \mathcal{L}_{u l, \rho}^{r}\left(\mathbb{R}^{N}\right)$ and Lemma 2.2. Moreover, we have

$$
\begin{aligned}
\| u(t) & -e^{t \Delta} u_{0} \|_{L_{u l, \rho}^{r}\left(\mathbb{R}^{N}\right)} \\
& \leq \int_{0}^{t}\left\|e^{(t-s) \Delta}|u(s)|^{p}\right\|_{L_{u l, \rho}^{r}\left(\mathbb{R}^{N}\right)} d s \\
& \lesssim \int_{0}^{t}\left(\rho^{-N\left(\frac{p}{q}-\frac{1}{r}\right)}+(t-s)^{-\frac{N}{2}\left(\frac{p}{q}-\frac{1}{r}\right)}\right) s^{-\frac{N}{2}\left(\frac{1}{r}-\frac{1}{q}\right) p} d s \cdot \sup _{0<s<t} s^{\sigma p}\|u(s)\|_{L_{u l, \rho}^{q}\left(\mathbb{R}^{N}\right)}^{p} \\
& \lesssim\left(t^{1-\frac{N}{2}\left(\frac{1}{r}-\frac{1}{q}\right) p} \rho^{-N\left(\frac{p}{q}-\frac{1}{r}\right)}+1\right) \sup _{0<s<t} s^{\sigma p}\|u(s)\|_{L_{u l, \rho}^{q}\left(\mathbb{R}^{N}\right)}^{p} \rightarrow 0 \text { as } t \rightarrow 0,
\end{aligned}
$$

since $u \in K$ and

$$
-\frac{N}{2}\left(\frac{1}{r}-\frac{1}{q}\right) p>-1, \quad-\frac{N}{2}\left(\frac{p}{q}-\frac{1}{r}\right)-\frac{N}{2}\left(\frac{1}{r}-\frac{1}{q}\right) p=-1 .
$$

Thus we have (A.7), and so $u \in C\left([0, T) ; \mathcal{L}_{u l, \rho}^{r}\left(\mathbb{R}^{N}\right)\right)$.

Finally we prove that the fixed point $u \in C\left([0, T) ; L_{u l, \rho}^{r}\left(\mathbb{R}^{N}\right)\right)$ is smooth so is a classical solution of (A.1). To this end, it suffices to prove $u \in L_{l o c}^{\infty}\left((0, T) ; L^{\infty}\left(\mathbb{R}^{N}\right)\right)$. Let $n \in \mathbb{N}$ and choose $\left\{q_{n}\right\}$ such that

$$
q_{1}:=q, \quad q_{n+1} \geq q_{n} \text { with } \frac{N}{2}\left(\frac{p}{q_{n}}-\frac{1}{q_{n+1}}\right)<1 .
$$

A simple calculation shows that

$$
\frac{1}{q_{n}}>p^{n-1}\left(\frac{1}{q}-\frac{p}{r}\right)+\frac{p}{r}
$$

Since $\frac{1}{q}-\frac{p}{r}<\frac{1}{q}-\frac{1}{r}<0$, there exists $n_{0} \in \mathbb{N}$ such that $\frac{N p}{2 q_{n_{0}}-1}<1$. Then we redefine $q_{n_{0}}:=\infty$ and consider the sequence $\left\{q_{n}\right\}_{n=1}^{n_{0}}$. Now fix $\epsilon>0$. Since $u \in Y_{M, T}$, we have $u \in L^{\infty}\left(\left[\epsilon / n_{0}, T\right] ; L_{u l, \rho}^{q_{1}}\left(\mathbb{R}^{N}\right)\right)$ and

$$
u\left(t+\epsilon / n_{0}\right)=e^{t \Delta}\left(u\left(\epsilon / n_{0}\right)\right)+\int_{0}^{t} e^{(t-s) \Delta}\left|u\left(s+\epsilon / n_{0}\right)\right|^{p-1} u\left(s+\epsilon / n_{0}\right) d s,
$$

which implies that there exists a constant $C(\epsilon)>0$ such that

$$
\begin{aligned}
& \left\|u\left(t+\frac{\epsilon}{n_{0}}\right)\right\|_{L_{u l, \rho}^{q_{2}}\left(\mathbb{R}^{N}\right)} \\
& \qquad\left(\rho^{-N\left(\frac{1}{q_{2}}-\frac{1}{q_{1}}\right)}+t^{-\frac{N}{2}\left(\frac{1}{q_{2}}-\frac{1}{q_{1}}\right)}\right)\left\|u\left(\frac{\epsilon}{n_{0}}\right)\right\|_{L_{u l, \rho}^{q_{1}}\left(\mathbb{R}^{N}\right)} \\
& \quad+\int_{0}^{t}\left(\rho^{-N\left(\frac{p}{q_{1}}-\frac{1}{q_{2}}\right)}+(t-s)^{-\frac{N}{2}\left(\frac{p}{q_{1}}-\frac{1}{q_{2}}\right)}\right)\left\|u\left(\cdot+\frac{\epsilon}{n_{0}}\right)\right\|_{L_{u l, \rho}^{q_{1}\left(\mathbb{R}^{N}\right)}}^{p} d s \leq C(\epsilon)
\end{aligned}
$$

for all $t \in\left[\epsilon / n_{0}, T-\epsilon / n_{0}\right]$. This proves $u \in L^{\infty}\left(\left[2 \epsilon / n_{0}, T\right] ; L^{q_{2}}\left(\mathbb{R}^{N}\right)\right)$. Iterating above argument $n_{0}$ times, we obtain $u \in L^{\infty}\left([\epsilon, T] ; L^{q_{n_{0}}}\left(\mathbb{R}^{N}\right)\right)$. Since $q_{n_{0}}=\infty$ and $\epsilon>0$ is arbitrary, we have $u \in L_{l o c}^{\infty}\left((0, T) ; L^{\infty}\left(\mathbb{R}^{N}\right)\right)$. Thus we complete the proof of Proposition 2.3 .

Acknowledgments. This work was partially funded by JSPS KAKENHI (grant number $15 \mathrm{~K} 17573$ and 15K17575) and Sumitomo Fundation (grant number 140825). 


\section{References}

[1] D. Andreucci and E. DiBenedetto, On the Cauchy problem and initial traces for a class of evolution equations with strongly nonlinear sources, Ann. Scuola Norm. Sup. Pisa Cl. Sci. 18 (1991), 363-441.

[2] J. M. Arriera, A. Rodriguez-Bernal, J. W. Cholewa and T. Dlotko, Linear parabolic equations in locally uniform spaces, Math. Models Methods Appl. Sci. 14 (2004), 253-293.

[3] P. Baras and M. Pierre, Critère d'existence de solutions positives pour des équations semilinéaires non monotones, Ann. Inst. H. Poincaré Anal. Non Linéaire 2 (1985), 185-212.

[4] H. Brezis and T. Cazenave, A nonlinear heat equation with singular initial data, J. Anal. Math. 68 (1996), 277-304.

[5] Y. Fujishima, Blow-up set for a superlinear heat equation and pointedness of the initial data, Discrete Continuous Dynamical Systems A 34 (2014), 4617-4645.

[6] Y. Giga, Solutions for semilinear parabolic equations in $L^{p}$ and regularity of weak solutions of the Navier-Stokes system, J. Differential Equations 62 (1986), 415-421.

[7] J. Ginibre and G. Velo, The Cauchy problem in local spaces for the complex GinzburgLandau equation II. Contraction methods, Commun. Math. Phys. 187 (1997), 45-79.

[8] A. Haraux and F. B. Weissler, Nonuniqueness for a semilinear initial value problem, Indiana Univ. Math. J. 31 (1982), 167-189.

[9] S. Ibrahim, R. Jrad, M. Majdoub and T. Saanouni, Local well posedness of a 2D semilinear heat equation, Bull. Belg. Math. Soc. Simon Stevin 21 (2014), 535-551.

[10] N. Ioku, The Cauchy problem for heat equations with exponential nonlinearity, J. Differential Equations 251 (2011), 1172-1194.

[11] N. Ioku, B. Ruf and E. Terraneo, Existence, non-existence, and uniqueness for a heat equation with exponential nonlinearity in $\mathbb{R}^{N}$, Math. Phys. Anal. Geom. 18 (2015), 18:29.

[12] K. Ishige, T. Kawakami and M. Sierżęga, Supersolutions of parabolic systems with power nonlinearities, J. Differential Equations 260 (2016), 6084-6107.

[13] K. Ishige and R. Sato, Heat equation with a nonlinear boundary condition and uniformly local $L^{r}$ spaces, Discrete Continuous Dynamical Systems A 36, 2627-2652.

[14] O. A. Ladyzenskaja, N. S. Solonikov and N. N. Ural'ceva, "Linear and quasilinear equations of parabolic type", Amer. Math. Soc., Providence, 1968.

[15] R. Laister, J.C. Robinson, M. Sierżȩga and A. Vidal-López, A complete characterization of local existence of semilinear heat equations in Lebesgue spaces, Ann. Inst. H. Poincaré Anal. Non Linéaire, in press.

[16] Y. Maekawa and Y. Terasawa, The Navier-Stokes equations with initial data in uniformly local $L^{p}$ spaces, Differential Integral Equations 19 (2006), 369-400.

[17] M. Nakamura and T. Ozawa, Nonlinear Schrödinger equations in the Sobolev space of critical order, J. Funct. Anal. 155 (1998), 364-380.

[18] W.-M. Ni and P. Sacks, Singular behavior in nonlinear parabolic equations, Trans. Amer. Math. Soc. 287 (1985), 657-671. 
[19] P. Quittner and P. Souplet, "Superlinear Parabolic Problems, Blow-up, Global Existence and Steady States", Birkhäuser Advanced Texts: Basler Lehrbücher Birkhäuser Verlag, Basel, 2007.

[20] J. C. Robinson and M. Sierżȩga, Supersolutions for a class of semilinear heat equations, Rev. Mat. Complut. 26 (2013), 341-360.

[21] B. Ruf and E. Terraneo, The Cauchy problem for a semilinear heat equation with singular initial data, Progr. Nonlinear Differential Equations Appl. 50 (2002), 295-309.

[22] D. H. Sattinger, Monotone methods in nonlinear elliptic and parabolic boundary value problems, Indiana Univ. Math. J. 21 (1972), 979-1000.

[23] S. Snoussi, S. Tayachi and F. B. Weissler, Asymptotically self-similar global solutions of a semilinear parabolic equation with a nonlinear gradient term, Proc. Roy. Soc. Edinburgh Sect. A 129 (1999), 1291-1307.

[24] S. Tayachi, Forward self-similar solutions of a semilinear parabolic equation with a nonlinear gradient term, Differential Integral Equations 9 (1996), 1107-1117.

[25] E. Terraneo, Non-uniqueness for a critical non-linear heat equation, Comm. Partial Differential Equations 27 (2002), 185-218.

[26] F. B. Weissler, Local existence and nonexistence for semilinear parabolic equations in $L^{p}$, Indiana Univ. Math. J. 29 (1980), 79-102. 